University of Wollongong

Research Online

Faculty of Business - Papers (Archive)

Faculty of Business and Law

$1-1-2015$

Self-similar measures in multi-sector endogenous growth models

Davide La Torre

Universita degli Studi di Milano

Simone Marsiglio

University of Wollongong, simonem@uow.edu.au

Franklin Mendivil

Arcadia University

Fabio Privileggi

Universita Del Piemonte Orientale

Follow this and additional works at: https://ro.uow.edu.au/buspapers

Part of the Business Commons

Research Online is the open access institutional repository for the University of Wollongong. For further information contact the UOW Library: research-pubs@uow.edu.au 


\title{
Self-similar measures in multi-sector endogenous growth models
}

\begin{abstract}
We analyze two types of stochastic discrete time multi-sector endogenous growth models, namely a basic Uzawa-Lucas $(1965,1988)$ model and an extended three-sector version as in La Torre and Marsiglio (2010). As in the case of sustained growth the optimal dynamics of the state variables are not stationary, we focus on the dynamics of the capital ratio variables, and we show that, through appropriate logtransformations, they can be converted into affine iterated function systems converging to an invariant distribution supported on some (possibly fractal) compact set. This proves that also the steady state of endogenous growth models-i.e., the stochastic balanced growth path equilibrium-might have a fractal nature. We also provide some sufficient conditions under which the associated self-similar measures turn out to be either singular or absolutely continuous (for the three-sector model we only consider the singularity).
\end{abstract}

\section{Keywords}

measures, multi, sector, endogenous, growth, models, self, similar

Disciplines

Business

\section{Publication Details}

La Torre, D., Marsiglio, S., Mendivil, F. \& Privileggi, F. (2015). Self-similar measures in multi-sector endogenous growth models. Chaos, Solitons and Fractals, 79 40-56. 


\title{
Self-Similar Measures in Multi-Sector Endogenous Growth Models*
}

\author{
Davide La Torre ${ }^{\dagger} \quad$ Simone Marsiglio Franklin Mendivil $^{\ddagger}$ \\ Fabio Privileggi \\ Forthcoming in Chaos, Solitons \& Fractals
}

\begin{abstract}
We analyze two types of stochastic discrete time multi-sector endogenous growth models, namely a basic Uzawa-Lucas (1988) model and an extended three-sector version as in La Torre and Marsiglio (2010). As in the case of sustained growth the optimal dynamics of the state variables are not stationary, we focus on the dynamics of the capital ratio variables, and we show that, through appropriate log-transformations, they can be converted into affine iterated function systems converging to an invariant distribution supported on some (possibly fractal) compact set. This proves that also the steady state of endogenous growth modelsi.e., the stochastic balanced growth path equilibrium - might have a fractal nature. We also provide some sufficient conditions under which the associated self-similar measures turn out to be either singular or absolutely continuous (for the three-sector model we only consider the singularity).
\end{abstract}

Keywords: Multi-Sector Growth Models, Endogenous Growth, Fractal, Self-Similar Measure, Singular and Absolutely Continuous Measure

JEL Classification: C61, O41

\section{Introduction}

Almost thirty years after the seminal work of Boldrin and Montrucchio (1986), it is now well known that also traditional (macro)economic models may give rise to complicated dynamics, including random dynamics eventually converging to (possibly singular) invariant measures supported on fractal sets. Montrucchio and Privileggi (1999) borrowing from the iterated function system (IFS)

${ }^{*}$ We wish to thank L. Gardini, V. Böhm and the participants in the $8^{t h}$ Workshop on Dynamic Models in Economics and Finance - MDEF 2014 (Urbino, Italy, September 2014) for insightful and constructive discussion. Also, NSERC is gratefully acknowledged for support through grant number 238549-2012.

${ }^{\dagger}$ University of Milan, Department of Economics, Management and Quantitative Methods, via Conservatorio 7, 20122 Milan, Italy, and Khalifa University, Department of Applied Mathematics and Sciences, PO Box 127788, Abu Dhabi, UAE, davide.latorre@unimi.it, davide.latorre@kustar.ac.ae,

† University of Wollongong, School of Accounting, Economics and Finance, Northfields Avenue, Wollongong 2522 NSW, Australia. simonem@uow.edu.au.

$\S^{\S}$ Acadia University, Department of Mathematics and Statistics, 2011 Acadia University, Wolfville, Nova Scotia, Canada, franklin.mendivil@acadiau.ca.

๑ University of Turin, Department of Economics and Statistics "Cognetti de Martiis", Lungo Dora Siena 100A, 10153, Turin, Italy, fabio.privileggi@unito.it. 
literature (Hutchinson, 1981; Barnsley, 1989; Vrscay, 1991) firstly show that standard stochastic economic growth models may show optimal dynamics defined by an IFS. The traditional one-sector growth model with Cobb-Douglas production and logarithmic utility has been extensively studied later. Mitra et al. (2003) show that its optimal path converges to a singular measure supported on a Cantor set, characterizing singularity versus absolute continuity of the invariant probability in terms of the parameters' values. Mitra and Privileggi (2004, 2006, 2009) further generalize the model and provide also an estimate of the Lipschitz constant for the maps of the optimal policy defined by an IFS 1 Only recently, the analysis has been extended in order to consider two-sector growth models, nowadays predominant in economic growth theory. La Torre et al. (2011) show that in a two-sector model with physical and human capital accumulation the optimal dynamics for the state variables can be converted through an appropriate log-transformation into an IFS converging to an invariant measure supported on a generalized Sierpinski gasket.

The aim of this paper it to further extend the analysis of fractal outcomes in optimal economic growth models by studying the behavior of multi-sector endogenous growth models. Indeed, thus far the focus has always been placed on neoclassical growth models, in which at steady state the economic growth rate is null, and nothing has been said on whether also a perpetually growing economy (i.e., an economy experiencing a strictly positive steady state growth rate) may achieve a fractal-type steady state. We thus analyze two alternative models of endogenous growth, specifically a two-sector and a three-sector model, based on the Uzawa-Lucas (1988) and La Torre and Marsiglio (2010) models, respectively. We show that even whenever perpetual growth is admissible the economy may develop along a (stochastic) balanced growth path equilibrium characterized by a fractal nature. However, since in such a framework the optimal dynamics of (physical, human and technological) capital are not stationary, we consider the dynamics of the capital ratio variables (specifically, the physical to human capital and technological to human capital ratios) and show that, through appropriate log-transformations, they can be converted into affine IFS converging to some distribution supported on a compact set which may be a fractal.2 We then also provide some sufficient conditions under which the associated self-similar measures turn out to be singular and absolutely continuous.

The paper proceeds as follows. In Section 2 the main results from the IFS theory that we will need in our analysis are briefly recalled and novel sufficient conditions (Theorem 5) for singularity of the invariant distribution are provided for a special class of two-dimensional affine IFS. In Section 3 we consider the simplest form of multi-sector endogenous growth models, namely a Uzawa-Lucas (1988) model driven by human capital accumulation. In Section 4 we analyze an extended version of the model, that is a three-sector model, as in La Torre and Marsiglio (2010), in which human capital is endogenously allocated across three (physical, human and knowledge) sectors. For both the models, we derive the optimal dynamics and construct an affine IFS conjugate to the optimal dynamics of stationary variables (the physical to human capital, and, in the latter, also the knowledge to human capital ratios). We provide, directly in terms of parameters of the model, sufficient conditions for the attractor of this conjugate IFS to be a fractal set (the Cantor

\footnotetext{
${ }^{1}$ Other recent applications of the IFS theory showing that some economic growth model converge to an invariant distribution supported on a Cantor set are Marsiglio (2012) and Privileggi and Marsiglio (2013). Specifically, Marsiglio (2012) analyzes a two-sector Solow model, while Privileggi and Marsiglio (2013) consider the sustainability problem in a stochastic economy-environment model.

${ }^{2}$ The advantage of introducing such a log-transformation consists of obtaining an affine system topologically conjugate to the original nonlinear system which allows to exploit the mathematical theory on IFS, thus simplifying the characterization of existence and uniqueness of (stochastic) fixed points. Without such a transformation, we would need to rely on more cumbersome ad-hoc approaches, like analyzing the eventual monotonicity properties of the optimal policies and dynamics, as, e.g., Brock and Mirman (1972) did in their seminal work.
} 
set for the two-sector model and a generalized Sierpinski gasket for the three-sector model). We also identify sufficient conditions under which the self-similar measures turn out to be singular and absolutely continuous. In Section 5 we build some examples of attractors, while Section 6 presents concluding remarks and proposes directions for future research.

\section{Iterated Function Systems}

An Iterated Function Systems (IFS) is a finite collection of contractive maps which are defined on a complete metric space. This collection of maps allows to formalize the notion of self-similarity and the definition of invariant set or attractor of the IFS. An Iterated Function System with Probabilities (IFSP), instead, consists of the above collection of IFS maps together with an associated set of probabilities. An IFSP induces a Markov operator on the set of all Borel probability measures and a notion of self-similar invariant measure. More details on these can be found in the fundamental works by Hutchinson (1981) and Barnsley and Demko (1985). Applications of these methods are in image compression, approximation theory, signal analysis, denoising, and density estimation (Freiberg et al., 2011; Kunze et al., 2007; Iacus and La Torre, 2005a, 2005b; La Torre et al., 2006, 2009; La Torre and Mendivil, 2008, 2009; La Torre and Vrscay, 2009; Mendivil and Vrscay, 2002a, 2002b). Now we recall, without proofs, some well known basic properties that will be used in the next sections.

We briefly introduce the notion of Hausdorff measure and Hausdorff dimension (more details can be found in Barnsley, 1989). Let $(X, d)$ be a metric space and let diam $(E)$ denote the diameter of a subset $E$ of $X$. Let $s \geq 0$ and $\delta>0$, and define

$$
\mathcal{H}_{\delta}^{s}(E)=\inf \left\{\sum_{k=1}^{\infty}\left[\operatorname{diam}\left(E_{k}\right)\right]^{s}: E \subset \bigcup_{i=1}^{\infty} E_{k}, \operatorname{diam}\left(E_{k}\right)<\delta\right\} .
$$

Now let us define

$$
\mathcal{H}^{s}(E)=\sup _{\delta>0} \mathcal{H}_{\delta}^{s}(E)
$$

Definition $1 \mathcal{H}^{s}(E)$ in (11) is called the s-dimensional Hausdorff measure. Furthermore, there exists a unique number $s_{0} \geq 0$ such that $\mathcal{H}^{s}(E)=\infty$ for $0 \leq s<s_{0}$ and $\mathcal{H}^{s}(E)=0$ for $s>s_{0}$. The number $s_{0}$ is called the Hausdorff dimension of $E$ and it is denoted by $\operatorname{dim}_{H}(E)$.

In what follows, let $(X, d)$ be a complete metric space and $w=\left\{w_{0}, \ldots, w_{m-1}\right\}$ a set of $m$ injective contraction maps $w_{i}: X \rightarrow X$, to be referred to as an m-map IFS. Let $0<\lambda_{i}<1$ denote the contraction factors of $w_{i}$ and define $\lambda:=\max _{i \in\{0, \ldots, m-1\}} \lambda_{i}$; clearly $0<\lambda<1$. Associated with the IFS mappings $w_{0}, \ldots, w_{m-1}$ there is a set-valued mapping $\hat{w}: \mathcal{K}(X) \rightarrow \mathcal{K}(X)$ defined over the space $\mathcal{K}(X)$ of all non-empty compact sets in $X$ as

$$
\hat{w}(A):=\bigcup_{i=0}^{m-1} w_{i}(A), \quad \forall A \in \mathcal{K}(X),
$$

where $w_{i}(A)=\left\{w_{i}(x): x \in A\right\}$ is the image of $A$ under $w_{i}, i=0,1, \ldots, m-1$. Let $\hat{w}^{t}(A)=$ $\hat{w}\left[\hat{w}^{t-1}(A)\right]$ for all $t \geq 1$, with $\hat{w}^{0}(A)=A$. The Hausdorff distance $d_{H}$ is defined as

$$
d_{H}(A, B)=\max \left\{\sup _{x \in A} \inf _{y \in B} d(x, y), \sup _{x \in B} \inf _{y \in A} d(x, y)\right\}, \quad \forall A, B \in \mathcal{K}(X) .
$$


Theorem 1 (Hutchinson, 1981) $\left(\mathcal{K}(X), d_{H}\right)$ is a complete metric space and $\hat{w}$ is a contraction mapping on $\left(\mathcal{K}(X), d_{H}\right)$ :

$$
d_{H}(\hat{w}(A), \hat{w}(B)) \leq \lambda d_{H}(A, B), \quad \forall A, B \in \mathcal{K}(X) .
$$

Therefore, there exists a unique set $A^{*} \in \mathcal{K}(X)$, such that $\hat{w}\left(A^{*}\right)=A^{*}$, the so-called attractor (or invariant set) of the IFS $\hat{w}$. Moreover, for any $A \in \mathcal{K}(X), d_{H}\left(\hat{w}^{t}(A), A^{*}\right) \rightarrow 0$ as $t \rightarrow \infty$.

\subsection{Invariant Measures}

Let $p=\left(p_{0}, p_{1}, \ldots, p_{m-1}\right), 0<p_{i}<1, i=0,1, \ldots, m-1$, be a partition of unity associated with the IFS mappings $w_{i}$, so that $\sum_{i=0}^{m-1} p_{i}=1$ (each $p_{i}$ represents a probability value attached to $w_{i}$ ). Let $\mathcal{M}(X)$ be the space of probability measures defined on the $\sigma$-algebra $\mathcal{B}(X)$ of Borel measurable subsets of $X$ and define for some $a \in X$ the set $\mathcal{M}_{1}(X)=\left\{\mu \in \mathcal{M}(X): \int_{X} d(a, x) \mathrm{d} \mu(x)<\infty\right\}$. Define the Monge-Kantorovich distance as

$$
d_{M}(\mu, \nu)=\sup \left\{\int_{X} f \mathrm{~d}(\mu-\nu): f \in \operatorname{Lip}_{1}(X)\right\}, \quad \forall \mu, \nu \in \mathcal{M}_{1}(X),
$$

where $\operatorname{Lip}_{1}(X)$ is the set of all Lipschitz functions on $X$ with Lipschitz constant equal to 1 . Associated with the IFS $(w ; p)$ is the so-called Markov operator, $M: \mathcal{M}_{1}(X) \rightarrow \mathcal{M}_{1}(X)$, defined as

$$
(M \mu)(A)=\sum_{i=0}^{m-1} p_{i} \mu\left[w_{i}^{-1}(A)\right], \quad \forall A \in \mathcal{B}(X),
$$

where $w_{i}^{-1}(S)=\left\{y \in X: w_{i}(y) \in S\right\}$. Let $M^{t} \mu=M\left(M^{t-1} \mu\right)$ for all $t \geq 1$, with $M^{0} \mu=\mu$.

Theorem 2 (Barnsley et al., 2008) If $X$ is a separable complete metric space $\left(\mathcal{M}_{1}(X), d_{M}\right)$ is a complete metric space; furthermore, if $X$ is compact, then $\mathcal{M}(X)=\mathcal{M}_{1}(X)$ and both are compact metric spaces under $d_{M}$.

$M$ is a contraction mapping on $\left(\mathcal{M}_{1}(X), d_{M}\right)$, specifically,

$$
d_{M}(M \mu, M \nu) \leq\left(\sum_{i} p_{i} \lambda_{i}\right) d_{M}(\mu, \nu), \quad \forall \mu, \nu \in \mathcal{M}_{1}(X),
$$

and thus there exists a unique probability measure $\mu^{*} \in \mathcal{M}_{1}(X)$, called invariant measure of the $\operatorname{IFS}(w ; p)$, such that $M \mu^{*}=\mu^{*}$.

For any $\mu \in \mathcal{M}_{1}(X), d_{M}\left(M^{t} \mu, \mu^{*}\right) \rightarrow 0$ as $t \rightarrow \infty$, which implies that $M^{t} \mu$ converges in the Monge-Kantorovich metric to $\mu^{*}$.

If the contraction mappings $w_{i}$ are assumed to be similitudes, i.e., if there exist numbers $0<\lambda_{i}<1$ such that

$$
d\left(w_{i}(x), w_{i}(y)\right)=\lambda_{i} d(x, y), \quad \forall x, y \in X, \quad i=0, \ldots, m-1,
$$

the attractor $A^{*}$ and the invariant measure $\mu^{*}$ are both said to be self-similar. As it is common in the IFS literature, however, in what follows we shall refer to any invariant measure $\mu^{*}$ generated by some IFS as 'self-similar'. 


\subsection{Absolutely Continuous vs. Singular Self-similar Measures}

It is known that the self-similar invariant measure $\mu^{*}$ determined by a IFS $(w ; p)$ generated by similitudes can be either absolutely continuous or singular with respect to the $n$-dimensional Lebesgue measure, according to the following definitions.

Definition 2 Two positive measures $\mu$ and $\nu$ defined on a measurable space $(\Omega, \Sigma)$ are called singular if there exist two disjoint sets $A$ and $B$ in $\Sigma$ whose union is $\Omega$ such that $\mu$ is zero on all measurable subsets of $B$ while $\nu$ is zero on all measurable subsets of $A$. This is denoted by $\mu \perp \nu$.

Definition 3 If $\mu$ and $\nu$ are two measures defined on a measurable space $(\Omega, \Sigma)$, we say that $\nu$ is absolutely continuous with respect to $\mu$ if $\nu(A)=0$ for any $A \in \Sigma$ such that $\mu(A)=0$. The absolute continuity of $\nu$ with respect to $\mu$ is denoted by $\nu \ll \mu$.

This distinction is crucial as in the former case $\mu^{*}$ can be represented by a density depending on some parameters, while in the latter case there is no simple way to represent it - one actually has to list all its values on every point in its support. The mathematical literature so far has dealt with this issue by trying to characterize absolute continuity vs. singularity of $\mu^{*}$ in terms of the parameters characterizing the IFS $(w ; p)$, specifically, in terms of $\lambda_{i}$ s and $p_{i}$ s configurations.

Especially the one-dimensional 2-maps IFS $(\lambda x, \lambda x+(1-\lambda) ; p,(1-p))$, with $0<\lambda<1$ and $0<p<1$, characterized by the same contraction factor $\lambda$ in both maps, has received much attention since the first half of the twentieth century, as its invariant measure $\mu^{*}$ is the same as that of the Erdös series $\sum_{s=0}^{\infty} \pm \lambda^{s}$ [it being understood that the minus sign is taken with probability $p$ and the plus sign with probability $(1-p)]$ translated over the interval $[0,1]$ (see Mitra et al., 2003). For $p=1 / 2$ the topic is known as the study of "symmetric infinite Bernoulli convolutions"; an exhaustive survey on the whole history of this subject can be found in Peres et al. (2000). It is straightforward to see that for $0<\lambda<1 / 2$ and any $0<p<1$ the support of $\mu^{*}$ is a Cantor set of Lebesgue measure zero, so that $\mu^{*}$ must be singular, while when $\lambda=1 / 2$ and $p=1 / 2 \mu^{*}$ turns out to be the uniform (Lebesgue) measure over $[0,1]$, which is clearly absolutely continuous. More complex, and hitherto incomplete, is the analysis for parameter values $1 / 2 \leq \lambda<1$ and $0<p<1$, for which the support of $\mu^{*}$ is the 'full' interval $[0,1]$. Solomyak (1995) made a real breakthrough when he established that, when $p=1 / 2$, for almost every $1 / 2<\lambda<1 \mu^{*}$ has density in $L^{2}(\mathbb{R})$ and for almost every $2^{-1 / 2}<\lambda<1$ the density is bounded and continuous (see also Peres and Solomyak, 1996, and Peres and Schlag, 2000). To the best of our knowledge, the contribution by Peres and Solomyak (1998), established for a generic family of contracting similitudes on the real line -including the general case $1 / 2 \leq \lambda<1$ and $0<p<1$ for the 2-maps IFS above - is still the most advanced available in the literature and proves useful for our purposes in the next Section 3 . We summarize below their results, together with the simpler cases discussed before, on the basis of the analysis in Mitra et al. (2003), so to provide an overall picture of the state of the art.

Theorem 3 Consider the 2-maps IFS $(\lambda x, \lambda x+(1-\lambda) ; p,(1-p))$ on $[0,1]$, with $0<\lambda<1$ and $0<p<1$, and let $A^{*}, \mu^{*}$ be its corresponding self-similar attractor and measure respectively.

1. For any $0<\lambda<1 / 2$ and $0<p<1 A^{*}$ is a Cantor set and $\mu^{*}$ is singular.

2. If $\lambda=1 / 2$, then $A^{*}$ is the full interval $[0,1]$ and

(a) if $p \neq 1 / 2$, then $\mu^{*}$ is singular,

(b) if $p=1 / 2$, then $\mu^{*}$ is absolutely continuous - it is the uniform (Lebesgue) measure over $[0,1]$. 
3. If $1 / 2<\lambda<1$ again $A^{*}$ is the full interval $[0,1]$ and

(a) if $1 / 2<\lambda<p^{p}(1-p)^{1-p}$ (the entropy curve), then $\mu^{*}$ is singular,

(b) (Peres and Solomyak, 1998) if $1 / 3 \leq p \leq 2 / 3$, then $\mu^{*}$ is absolutely continuous for Lebesgue a.e. $p^{p}(1-p)^{1-p}<\lambda<1$,

(c) (Peres and Solomyak, 1998) if $0.156<p<1 / 3$ or $2 / 3<p<0.844$, then $\mu^{*}$ is absolutely continuous for Lebesgue a.e. $p^{p}(1-p)^{1-p}<\lambda<0.649$, while, for any $1<\gamma \leq 2$ such that $\left[p^{\gamma}+(1-p)^{\gamma}\right]^{1 /(\gamma-1)}<0.649$, $\mu^{*}$ has density in $L^{\gamma}$ for Lebesgue a.e. $\left[p^{\gamma}+(1-p)^{\gamma}\right]^{1 /(\gamma-1)} \leq \lambda<0.649$.

Theorem 3 still leaves room for further research, as the parameter configurations $0<p<1 / 3$ and $2 / 3<p<1$ for $0.649 \leq \lambda<1$ remain unsolved. Figure 5 in Mitra et al. (2003) draws a bifurcation diagram reporting all cases described in Theorem 3 .

Only little progress has been made after the results just stated. It is worth mentioning Niu and Xi (2007) who establish singularity of the self-similar measure for very peculiar IFS with $m$ maps that are similitudes on the real line. Here we report the following result, proved in Ngai and Wang (2005), that generalize some of the results of Theorem 3 to $m$-maps IFS on $\mathbb{R}^{n}$ having similitudes characterized by different contraction factors $0<\lambda_{i}<1$, namely

$$
w_{i}(x)=\lambda_{i} Q_{i} x+\xi_{i}, \quad i=0, \ldots, m-1,
$$

where $Q_{i}$ is a $n \times n$ orthogonal matrix and $\xi_{i} \in \mathbb{R}^{n}$ for each $i$. We denote by $A^{*} \subset \mathbb{R}^{n}$ the corresponding attractor and by $\mathcal{L}^{n}$ the $n$-dimensional Lebesgue measure.

Definition 4 The IFS satisfies the open set condition (OSC) if there exists a nonempty open set $U$ such that $w_{i}(U) \subset U$ for all $i=0, \ldots, m-1$ and $w_{i}(U) \cap w_{j}(U)=\varnothing$ for all $i \neq j$.

Theorem 4 (Ngai and Wang, 2005) Let $(w ; p)$ be an IFS on $\mathbb{R}^{n}$ with maps $w_{i}: \mathbb{R}^{n} \rightarrow \mathbb{R}^{n}$ defined by (4) and let $p=\left(p_{0}, p_{1}, \ldots, p_{m-1}\right)$ be the associated probability weights. Denote by $\mu^{*}$ the self-similar invariant measure supported over $A^{*}$ defined by $(w ; p)$.

i) If $\prod_{i=0}^{m-1} p_{i}^{p_{i}} \lambda_{i}^{-n p_{i}}>1$, then $\mu^{*}$ is singular.

ii) If $\prod_{i=0}^{m-1} p_{i}^{p_{i}} \lambda_{i}^{-n p_{i}}=1$ but $p_{i} \neq \lambda_{i}^{n}$ for some $i$, then $\mu^{*}$ is singular.

iii) If $p_{i}=\lambda_{i}^{n}$ for all $i=0, \ldots, m-1$, then $\mu^{*}$ is absolutely continuous if and only if the IFS $(w ; p)$ satisfies the open set condition $(O S C)$. In this case $\mu^{*}=\left.\delta \mathcal{L}^{n}\right|_{A^{*}}$, where $A^{*}$ is the attractor of the IFS, and $\delta=1 / \mathcal{L}^{n}\left(A^{*}\right)$; that is, $\mu^{*}$ is the uniform (n-dimensional Lebesgue) measure over $A^{*} \subset \mathbb{R}^{n}$.

Case (i) generalizes cases 1, 2a and 3a of Theorem 3 altogether to IFS with any number of similitudes on $\mathbb{R}^{n}$ with different contraction factors $\lambda_{i}$; case (ii) actually adds an important piece of information by extending the conclusion in $3 \mathrm{a}$ of Theorem 3 to the generalized boundary (entropy) curve corresponding to $\lambda=p^{p}(1-p)^{1-p}$ there; finally, case (iii) generalizes case $2 \mathrm{~b}$ of Theorem 3. Indeed, in the latter scenario the OSC requires that the image sets of the similitudes of the attractor, $w_{i}\left(A^{*}\right)$, have only "small overlap" (sometimes called "just touching"), which-because in Theorem 3 the two maps are assumed to have both the same slope, $\lambda_{0}=\lambda_{1}=\lambda$-for the IFS $(\lambda x, \lambda x+(1-\lambda) ; p,(1-p))$ translates into $\lambda=p=1 / 2$, which implies that $A^{*}$ is the full interval $[0,1]$ and the image sets are equal to $w_{0}([0,1])=[0,1 / 2]$ and $w_{1}([0,1])=[1 / 2,1]$, having only 
the unique point $x=1 / 2$ in common (see Schief, 1994). Theorem 1.2 in Ngai and Wang (2005) to some extent generalizes results $3 \mathrm{~b}$ and $3 \mathrm{c}$ of Theorem 3 to IFS with any number of similitudes with different contraction factors, however only for maps on the real line.

We end this section providing our own novel contribution to the latter literature with the next Theorem 5, which considers the special case of an affine three-map IFS on $X=R^{2}$ with constant linear parts given by the upper triangular matrix

$$
Q=\left[\begin{array}{ll}
a & c \\
0 & b
\end{array}\right]
$$

This type of IFS will become relevant in Section 4, where a three-sector model giving rise to an affine IFS of this type [see (34) and (35)] will be thoroughly analyzed.

There are not many results known about this situation, here we indicate a simple sufficient condition for singularity of the invariant measure. The simplest case is when the attractor of the IFS (the support of $\mu^{*}$ ) has dimension strictly less than 2, in which case it is obviously singular; so we start with this case. Define the function $\Phi(s)$ by

$$
\Phi(s)= \begin{cases}\max \left\{b^{s}, a^{s}\right\} & \text { if } s \leq 1 \\ \max \left\{a b^{s-1}, b a^{s-1}\right\} & \text { if } 1<s \leq 2 .\end{cases}
$$

Then by results of Falconer and Miao (2007), an upper bound for the dimension $\operatorname{dim}_{H}$ of the attractor $A^{*}$ of this IFS is given by

$$
\operatorname{dim}_{H}\left(A^{*}\right) \leq \min \left\{2, \Phi^{-1}\left(\frac{1}{3}\right)\right\} .
$$

It can be shown that $\Phi$ is a strictly decreasing function of $s$ and thus there is a unique $s$ such that $\Phi(s)=1 / 3$. Assuming that $a \leq b$, we have $a^{s} \leq b^{s}$ for all $s$ and $a b^{s-1} \leq b a^{s-1}$ for $s \leq 2$. Thus, under $a \leq b$ we have

$$
\Phi(s)= \begin{cases}b^{s} & \text { if } 0 \leq s \leq 1 \\ b a^{s-1} & \text { if } 1<s \leq 2\end{cases}
$$

Thus, we have either

$$
\operatorname{dim}_{H}\left(A^{*}\right) \leq-\frac{\ln 3}{\ln b} \quad \text { or } \quad \operatorname{dim}_{H}\left(A^{*}\right) \leq \frac{\ln [a /(3 b)]}{\ln a} .
$$

Therefore, $\operatorname{dim}_{H}\left(A^{*}\right)<2$ if $a b<1 / 3$.

The results in Falconer and Miao (2007) require the IFS to satisfy the OSC (Definition 4 above) in order for the dimension result to be exact. Hence, it is also possible that the dimension is less than 2 for a larger range of parameters than that given by $a b<1 / 3$.

The above (dimension-based) result can be extended by proving the following Theorem 5 , which can be thought of as an analogue of Theorem 3a or Theorem 4i. The strategy of the proof is the same as Theorem 4, but we include it because we use the entropy in an essentially different way. In the sequel we will denote by $\{0,1,2\}^{\mathbb{N}}$ the collection of infinite words in the alphabet $\{0,1,2\}$, by $\{0,1,2\}^{n}$ the collection of words of length $n$, and by $L^{2}$ the 2 -dimensional Lebesgue measure.

Theorem 5 Let $(w ; p)$ be an IFS on $\mathbb{R}^{2}$ having maps $w_{i}: \mathbb{R}^{2} \rightarrow \mathbb{R}^{2}$ defined by $w_{i}(x)=Q x+\xi_{i}$, with $Q$ as in (5), $\xi_{i} \in \mathbb{R}^{2}$ for $i=0,1,2$, and let $p=\left(p_{0}, p_{1}, p_{2}\right)$ be the associated probability weights. If $a b<p_{0}^{p_{0}} p_{1}^{p_{1}} p_{2}^{p_{2}}$, the invariant measure $\mu^{*}$ defined by $(w ; p)$ is singular. 
Proof. For $\sigma \in\{0,1,2\}^{\mathbb{N}}$ and for $n \in \mathbb{N}, i=0,1,2$, define

$$
\sigma(n, i)=\#\left\{j \leq n: \sigma_{j}=i\right\}
$$

Fix $k \in \mathbb{N}$ and consider the set

$$
S_{n}=\left\{\sigma \in\{0,1,2\}^{n}:\left|\frac{\sigma(n, i)-n p_{i}}{\sqrt{n} \sqrt{p_{i}\left(1-p_{i}\right)}}\right| \leq k, i=0,1,2\right\}
$$

so that $S_{n}$ is the set of $k$-typical sequences of length $n$. Then from Chebyshev's inequality we have

$$
\operatorname{Pr}\left(S_{n}\right) \geq 1-\frac{3}{k^{2}}
$$

which is independent of $n$. Furthermore, by Theorem 1.3.4 in Roman (1992) we have

$$
\# S_{n} \leq\left(p_{0}^{p_{0}} p_{1}^{p_{1}} p_{2}^{p_{2}}\right)^{-n} 3^{C \sqrt{n}}
$$

for some constant $C>0$. For notational ease define

$$
w_{\sigma}=w_{\sigma_{1}} \circ w_{\sigma_{2}} \circ \cdots \circ w_{\sigma_{n}}
$$

as the $n$-fold composition given by $\sigma \in\{0,1,2\}^{n}$. Now, let

$$
J_{n}=\bigcup_{\sigma \in S_{n}} w_{\sigma}(K) \subset K
$$

Then $\mu\left(J_{n}\right)=\operatorname{Pr}\left(S_{n}\right) \geq 1-3 / k^{2}$ uniformly in $n$ and

$$
\begin{aligned}
\mathcal{L}^{2}\left(J_{n}\right) & \leq \sum_{\sigma \in S_{n}} \mathcal{L}^{2}\left[w_{\sigma}(K)\right]=\mathcal{L}^{2}(K) \sum_{\sigma \in S_{n}}(a b)^{n}=\mathcal{L}^{2}(K)\left(\# S_{n}\right)(a b)^{n} \\
& \leq \mathcal{L}^{2}(K)\left(\frac{a b}{p_{0}^{p_{0}} p_{1}^{p_{1}} p_{2}^{p_{2}}}\right)^{n} 3^{C \sqrt{n}} \rightarrow 0
\end{aligned}
$$

as $n \rightarrow \infty$. Thus, $\mu^{*} \perp \mathcal{L}^{2}$.

Notice that the minimum value of $p_{0}^{p_{0}} p_{1}^{p_{1}} p_{2}^{p_{2}}$ is $1 / 3$ and occurs when $p_{0}=p_{1}=p_{2}=1 / 3$. Thus if $a b<1 / 3 \mu^{*}$ is singular for any choice of $p_{0}, p_{1}, p_{2}$ and so this result includes the dimension-based result previously mentioned.

Finally, Theorems 22 and 23 in Shmerkin (2006) imply that for any probabilities $p_{i}$ if the Lebesgue measure of $A^{*}$ is positive, then either $\mu^{*} \perp \mathcal{L}^{2}$ or we have both $\mu^{*} \ll \mathcal{L}^{2}$ and $\left.\mathcal{L}^{2}\right|_{A^{*}} \ll \mu^{*}$ (the two measures are mutually absolutely continuous). Specifically, as if $\mathcal{L}^{2}\left(A^{*}\right)=0$ we automatically have $\mu^{*} \perp \mathcal{L}^{2}$, this always implies that either $\mu^{*} \perp \mathcal{L}^{2}$ or they are mutually absolutely continuous.

\section{The Uzawa-Lucas Model}

We first study the Uzawa-Lucas (1988) model of endogenous growth where the social planner seeks to maximize social welfare subject to the physical and human capital constraints, choosing consumption, $c_{t}>0$, and the share of human capital employed in physical production, $0<u_{t}<1$. 
The welfare is the expected infinite discounted $(0<\beta<1$ is the pure rate of time preference) sum of instantaneous utilities [assumed to be logarithmic, $u\left(c_{t}\right)=\ln c_{t}$ ]. Physical capital, $k_{t}$, accumulates according to the difference between the production of the unique final good and consumption, $k_{t+1}=y_{t}-c_{t}$, where production is assumed to use a Cobb-Douglas technology combining physical and the allocated share of human capital, $y_{t}=k_{t}^{\alpha}\left(u_{t} h_{t}\right)^{1-\alpha}$, where $0<\alpha<1$ is the physical capital share and $u_{t}$ the proportion of human capital employed in physical production. The remaining share, $1-u_{t}$ is used to produce new human capital, $h_{t}$, according to a linear technology; thus, the law of motion of human capital is equal to: $h_{t+1}=b\left(1-u_{t}\right) h_{t}$, with $b>0$. However, the final sector is affected by exogenous perturbations, $z_{t}$, which enter multiplicatively the production function; these shocks are independent and identically distributed, and take on two values: $z_{t} \in\{q, 1\}$, where $0<q<1$. We assume that educational choices are not affected at all by eventual shocks. This means that at any time, given the realization of the random shocks, the economy may be in two alternative situations: i) an economic crisis due to a supply shock lowering physical productivity, corresponding to $z_{t}=q$, and ii) a business-as-usual scenario with no shocks in which the whole economy evolves along its full capacity, corresponding to $z_{t}=1$. These two alternative scenarios occur with (constant) probability $p$ and $1-p$, respectively.

The social planner problem consists of choosing the level of consumption and the shares of human capital to allocate to the physical sector in order to maximize social welfare, taking into account the dynamic evolution of physical and human capital, the presence of random shocks $z_{t} \in\{q, 1\}$, and the given initial conditions $k_{0}, h_{0}$, and $z_{0}$ :

$$
\begin{aligned}
& V\left(k_{0}, h_{0}, z_{0}\right)=\max _{\left\{c_{t}, u_{t}\right\}_{t=0}^{\infty}} \mathbb{E}_{0} \sum_{t=0}^{\infty} \beta^{t} \ln c_{t} \\
\text { s.t. } & \left\{\begin{array}{l}
k_{t+1}=z_{t} k_{t}^{\alpha}\left(u_{t} h_{t}\right)^{1-\alpha}-c_{t} \\
h_{t+1}=b\left(1-u_{t}\right) h_{t} \\
k_{0}>0, h_{0}>0, z_{0} \in\{q, 1\} \text { are given, }
\end{array}\right.
\end{aligned}
$$

where $\mathbb{E}_{0}$ denotes expectation at time $t=0$. The Bellman equation associated to (6) reads as:

$$
V\left(k_{t}, h_{t}, z_{t}\right)=\max _{c_{t}, u_{t}}\left[\ln c_{t}+\beta \mathbb{E}_{t} V\left(k_{t+1}, h_{t+1}, z_{t+1}\right)\right] .
$$

By applying the Verification principle, it is possible to obtain an analytical expression for the value function of the above problem, and consequently derive explicitly the optimal dynamics of physical and human capital (see also Bethmann, 2007, 2013).

Proposition 1 Given the problem in (6) under (7), the following results hold.

i) The Bellman equation (8) has solution given by:

$$
V\left(k_{t}, h_{t}, z_{t}\right)=\theta+\theta_{k} \ln k_{t}+\theta_{h} \ln h_{t}+\theta_{z} \ln z_{t},
$$

where $\theta_{j}, j \in\{k, h, z\}$, are defined as follows:

$$
\theta_{k}=\frac{\alpha}{1-\alpha \beta}, \quad \theta_{h}=\frac{1-\alpha}{(1-\alpha \beta)(1-\beta)}, \quad \theta_{z}=\frac{1}{1-\alpha \beta},
$$

and the constant term $\theta$ is given by:

$$
\begin{aligned}
\theta=\frac{1}{1-\beta}\left[\ln (1-\alpha \beta)+\frac{\alpha \beta}{1-\alpha \beta} \ln (\alpha \beta)+\right. & \frac{1-\alpha}{1-\alpha \beta} \ln (1-\beta) \\
& \left.\quad+\frac{(1-\alpha) \beta}{(1-\alpha \beta)(1-\beta)} \ln \beta+\frac{\beta}{(1-\alpha \beta)} \mathbb{E} \ln z\right] .
\end{aligned}
$$


ii) The optimal policy rules for consumption, $c_{t}$, and share of human capital allocated to physical production, $u_{t}$, are respectively given by:

$$
\begin{aligned}
& c_{t}=(1-\alpha \beta)(1-\beta)^{1-\alpha} z_{t} k_{t}^{\alpha} h_{t}^{1-\alpha} \\
& u_{t} \equiv 1-\beta \quad \forall t .
\end{aligned}
$$

The optimal dynamics of physical, $k_{t+1}$, and human, $h_{t+1}$, capital are the following:

$$
\begin{aligned}
& k_{t+1}=\alpha \beta(1-\beta)^{1-\alpha} z_{t} k_{t}^{\alpha} h_{t}^{1-\alpha} \\
& h_{t+1}=b \beta h_{t} .
\end{aligned}
$$

The proof of Proposition 1 parallels that of Proposition 1 in La Torre et al. (2011); hence, we omit the details.

The results highlighted in Proposition 1 are pretty standard in the literature (see Bethmann, 2007, 2013; La Torre et al., 2011). It is also very well-known that the Uzawa-Lucas (1988) framework, because of the linearity in the production of (new) human capital, may generate sustained long-run growth. Specifically, the system (9)-(10) is diverging whenever $b>1 / \beta$ as, in this case, both physical and human capital grow without any bound. Therefore, in such a scenario we need to introduce a change of variables in order to obtain a system converging to a stationary equilibrium. The natural candidate is the physical to human capital ratio, $\chi_{t}=k_{t} / h_{t}$, which, incidentally, reduces the two-dimensional optimal dynamics (9)-(10) into a one-dimensional dynamic. Indeed, the physical to human capital ratio $\chi_{t}$ evolves over time according to the following stochastic nonlinear difference equation:

$$
\chi_{t+1}=\frac{\alpha(1-\beta)^{1-\alpha}}{b} z_{t} \chi_{t}^{\alpha} .
$$

Denoting by $\sigma$ the linear coefficient in (11),

$$
\sigma=\frac{\alpha(1-\beta)^{1-\alpha}}{b},
$$

the nonlinear IFS associated to (11) defined by the two maps

$$
\left\{\begin{array}{l}
f_{0}(\chi)=\sigma q \chi^{\alpha} \quad \text { with probability } p \\
f_{1}(\chi)=\sigma \chi^{\alpha} \quad \text { with probability } 1-p
\end{array}\right.
$$

eventually traps the new variable $\chi$ into the compact interval $A=\left[(\sigma q)^{1 /(1-\alpha)}, \sigma^{1 /(1-\alpha)}\right]$, with endpoints corresponding to the fixed points of $f_{0}$ and $f_{1}$ respectively; such a scenario corresponds to growth rate values for the original variables, $k_{t}$ and $h_{t}$, oscillating between finite positive bounds. If the IFS (13) converges to some invariant measure supported over (possibly a fractal subset of) $A$, then we can interpret such a situation as a steady state representing a stochastic balanced growth path (SBGP) equilibrium, the stochastic equivalent of a typical equilibrium in deterministic endogenous growth theory.

Note that whenever $\alpha>q$ the IFS (13) turns out to be non-contractive, as there exists a right neighborhood of the left fixed point $(\sigma q)^{1 /(1-\alpha)}$ on which $f_{1}^{\prime}>13$ In this case, the results

\footnotetext{
${ }^{3}$ To see this, compute the derivative of the 'higher' map $f_{1}$ on the left fixed point, $(\sigma q)^{1 /(1-\alpha)}: f_{1}^{\prime}\left[(\sigma q)^{1 /(1-\alpha)}\right]=$ $\sigma \alpha\left[(\sigma q)^{1 /(1-\alpha)}\right]^{\alpha-1}=\sigma \alpha /(\sigma q)=\alpha / q$, which is clearly larger than 1 whenever $\alpha>q$. As $f_{0}^{\prime}\left[(\sigma q)^{1 /(1-\alpha)}\right]=\alpha<1$ and both $f_{0}$ and $f_{1}$ are strictly increasing and strictly concave, $\alpha / q$ is the Lipschitz constant of the IFS (13) over the trapping set $\left[(\sigma q)^{1 /(1-\alpha)}, \sigma^{1 /(1-\alpha)}\right]$.
} 
of Section 2, which provide only sufficient conditions for a (contractive) IFS to converge to a unique invariant measure, cannot be directly applied; however, the logarithmic transformation to the nonlinear dynamics in (11) contemplated by the next Proposition 2 allows us to establish the existence of a unique invariant measure for (13) indirectly. Such a transformation yields a conjugate affine system which can be represented by an IFS characterized by contractive similitudes.

Proposition 2 The one-to-one logarithmic transformation $\chi_{t} \rightarrow \varphi_{t}$ defined by:

$$
\varphi_{t}=-\frac{1-\alpha}{\ln q} \ln \chi_{t}+1+\frac{\ln \sigma}{\ln q}
$$

with $\sigma$ defined in (12), defines a contractive affine IFS equivalent to the nonlinear dynamics in (11) composed of two maps $w_{0}, w_{1}:[0,1] \rightarrow[0,1]$, where 0 and 1 are the fixed points of $w_{0}$ and $w_{1}$ respectively, given by:

$$
\begin{cases}w_{0}(\varphi)=\alpha \varphi & \text { with probability } p \\ w_{1}(\varphi)=\alpha \varphi+(1-\alpha) & \text { with probability } 1-p .\end{cases}
$$

The IFS (15) converges in the Monge-Kantorovich metric to a unique self-similar measure supported on a compact attractor which is either the interval $[0,1]$ when $1 / 2 \leq \alpha<1$ or a Cantor set when $0<\alpha<1 / 2$.

Proposition 2 follows immediately from Theorems 1 and 2 in Section 2 .

Note that the one-sector stochastic optimal growth model discussed in Mitra et al. (2003) exhibits the same optimal dynamics as in (15). Indeed, besides the different constant $\sigma$ as in (12), the dynamics described by (11) is the same as the optimal dynamics of capital in the one-sector growth model; hence, also the no-overlap condition $0<\alpha<1 / 2$ yielding a support which is a Cantor set is exactly the same. The novelty in our model is that here what converges to an invariant measure supported on a Cantor set is a transformation of the physical to human capital ratio (and not a transformation of physical capital); therefore, we have just shown that also an economy experiencing sustained growth can exhibit a long-run pattern related to some fractal attractor. Specifically, the SBGP equilibrium has a fractal nature whenever $\alpha<1 / 2$.

Note that Proposition 2 uses the physical capital share $0<\alpha<1$ as the contraction factor to establish convergence of (15). Because (14) is a one-to-one transformation of the nonlinear IFS (13), an immediate consequence of Proposition 2 is the following Corollary 1 establishing weak convergence of (13) to a unique invariant measure also when $\alpha>q$, that is, when it is non-contractive and falls outside the class of IFS considered in Section 2, We shall say that a set $A \subset \mathbb{R}$ is a generalized (topological) Cantor set if it is totally disconnected and perfect 4

Corollary 1 For any parameters' configuration such that $0<\alpha<1,0<\beta<1,0<q<1$, $0<p<1$, and $b>1 / \beta$, envisaging sustained growth for the stochastic discrete-time Uzawa-Lucas model, the nonlinear IFS (13) converges in the Monge-Kantorovich metric to a unique invariant measure supported either over the full interval $A^{*}=\left[(\sigma q)^{1 /(1-\alpha)}, \sigma^{1 /(1-\alpha)}\right]$ or over some compact subset of it. In the latter case, whenever $0<\alpha<1 / 2$ the attractor $A^{*}$ of (13) is a generalized topological Cantor set contained in $\left[(\sigma q)^{1 /(1-\alpha)}, \sigma^{1 /(1-\alpha)}\right]$.

\footnotetext{
${ }^{4}$ Recall that a set $A \subseteq X$, where $(X, d)$ is a metric space, is said to be totally disconnected if its only connected subsets are one-point sets: formally, for any two distinct points $x, y$ in $A$, there are two non-empty open disjoint sets $U$ and $V$ such that $x \in U, y \in V$ and $(U \cap A) \cup(V \cap A)=A$. A set $A \subseteq X$ is said to be perfect if it is equal to the set of its accumulation points; that is, it is a closed set which contains no isolated points.
} 
Proof. As there is a one-to-one correspondence between (11) and (14), the fist part is an immediate consequence of Proposition 2. To establish the second part, we must show that the no-overlap property for the IFS (13) is the same as the no-overlap property $0 \leq \alpha<1 / 2$ for the IFS (15): as both maps $f_{0}, f_{1}$ are strictly increasing, the former property (see Mitra and Privileggi, 2009) can be written as $f_{0}\left[\sigma^{1 /(1-\alpha)}\right]<f_{1}\left[(\sigma q)^{1 /(1-\alpha)}\right] \Longleftrightarrow \sigma q\left[\sigma^{1 /(1-\alpha)}\right]^{\alpha}<\sigma\left[(\sigma q)^{1 /(1-\alpha)}\right]^{\alpha} \Longleftrightarrow$ $q(\sigma)^{\alpha /(1-\alpha)}<(\sigma q)^{\alpha /(1-\alpha)} \Longleftrightarrow q<q^{\alpha /(1-\alpha)} \Longleftrightarrow q^{(2 \alpha-1) /(1-\alpha)}>1 \Longleftrightarrow \alpha<1 / 2$, establishing the result.

Corollary 1) states that, if $0<\alpha<1 / 2$, the attractor of the nonlinear IFS (13) is a distorted (due to nonlinearity of the maps in the IFS) Cantor set contained in the interval $\left[(\sigma q)^{1 /(1-\alpha)}, \sigma^{1 /(1-\alpha)}\right]$. Mitra and Privileggi (2004, 2006, 2009) dealt with nonlinear IFS generating optimal policies for the one-sector stochastic optimal growth model, starting from a class of the type in (13) and then increasing the degree of generality; however, all results established by the authors hold under assumptions on the primitives of the model guaranteeing that such IFS satisfy the contraction property, on which all results in Section 2 are based. Proposition 2, joint with Corollary 1 , expands these results outside the family of contractive IFS, at least for the specific functional forms chosen here. Clearly the logarithmic-Cobb-Douglas specification of our model plays a major role in allowing for the transformation (14) 5

Remark 1 The literature on IFS actually encompasses a more general result in which the assumption that the IFS mappings are contractions can be slightly relaxed and substituted with a broader notion of "contraction on average"; in this case existence and uniqueness of an invariant measure $\mu^{*}$ for the IFS can still be established (see, e.g., Proposition 5.1 in Diaconis and Friedman, 1999). Specifically, denoting by $\lambda_{i}$ the Lipschitz constant associated to the $i^{\text {th }}$ map of the IFS, for $i=0, \ldots, m-1$, the IFS is contracting on average if

$$
\sum_{i=0}^{m-1} p_{i} \ln \lambda_{i}<0
$$

in which smaller probability weights $p_{i}$ associated to steeper maps neutralize their effect when they are not contractions, i.e., when $\lambda_{i}>1$.

In our model the Lipschitz constants of the two maps in (13) correspond to the slopes of $f_{0}$ and $f_{1}$ on the smallest fixed point $(\sigma q)^{1 /(1-\alpha)}: \lambda_{0}=\alpha$ and $\lambda_{1}=\alpha / q$ respectively (see footnote 31). In this case (16) translates into $p \ln \alpha+(1-p) \ln (\alpha / q)=\ln \alpha-(1-p) \ln q<0$, which is equivalent to $\alpha / q<e^{1-p}$, clearly a more general assumption than the requirement for both maps to be contractive - corresponding to $\alpha / q<1$. However, the assumptions of Corollary 1 clearly include the case $\alpha / q \geq e^{1-p}$, stressing further its contribution outside the general theory.

Figure1 shows an example of parameters' configuration such that the IFS (13) is non-contractive, having Lipschitz constant, $\lambda_{1}$, associated to $f_{1}$ larger than 1 . Specifically, setting the fundamentals as $\alpha=1 / 3, q=1 / 6, p=2 / 3, \beta=0.96$ and $b=1.052$ (so that there is sustained growth as $b>1.042=1 / \beta)$, it turns out that $\sigma=0.037$ in (12), the fixed points of the two nonlinear

\footnotetext{
${ }^{5}$ Incidentally, we note that the same conclusion can be drawn for the one-sector stochastic optimal growth model in Mitra et al. (2003): the nonlinear IFS representing the optimal capital dynamics there converges weakly to a unique invariant measure also when the ratio physical capital share over the technological shock is larger than one, implying that the nonlinear IFS is not a contraction.
} 
maps $f_{0}, f_{1}$ are $\chi_{0}^{*}=(\sigma q)^{1 /(1-\alpha)}=0.0005$ and $\chi_{1}^{*}=0.007$ respectively, so that the trapping interval is $\left[\chi_{0}^{*}, \chi_{1}^{*}\right]=[0.0005,0.007]$, and their Lipschitz constants are $\lambda_{0}=f_{0}^{\prime}\left(\chi_{0}^{*}\right)=\alpha=1 / 3$ and $\lambda_{1}=f_{1}^{\prime}\left(\chi_{0}^{*}\right)=\alpha / q=2>e^{1-p}=1.4>1$; thus, the IFS is non-contractive, not even 'on average'. Moreover, as $\alpha=1 / 3<1 / 2$, in the figure it can be clearly seen that the images $f_{0}\left(\left[\chi_{0}^{*}, \chi_{1}^{*}\right]\right)$ and $f_{1}\left(\left[\chi_{0}^{*}, \chi_{1}^{*}\right]\right)$ do not overlap [because $\left.f_{0}\left(\chi_{1}^{*}\right)<f_{1}\left(\chi_{0}^{*}\right)\right]$, so that, according to Corollary 1, the attractor $A^{*}$ is a generalized topological Cantor set in $\left[\chi_{0}^{*}, \chi_{1}^{*}\right]$.

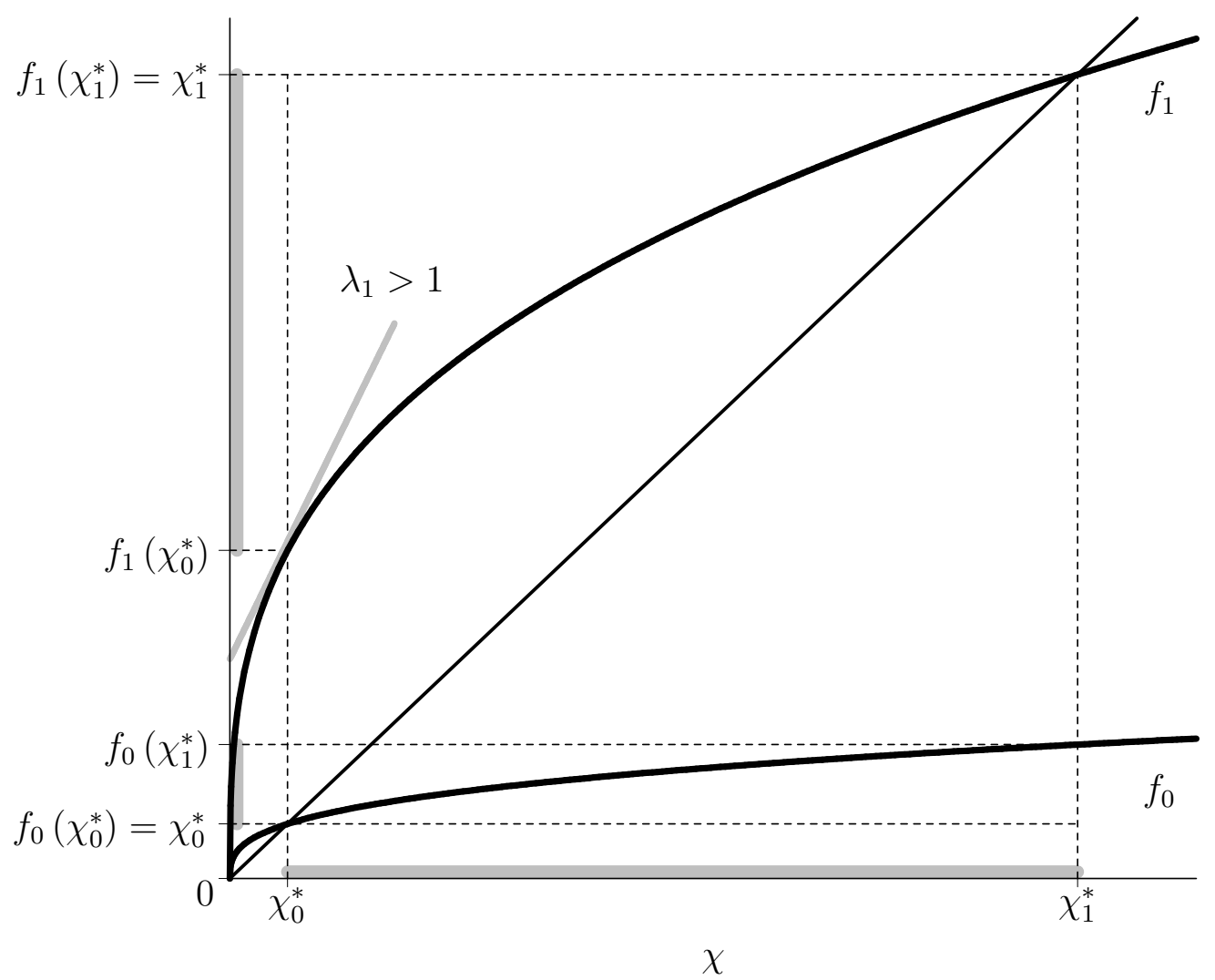

Figure 1: the nonlinear maps $f_{0}$ and $f_{1}$ in (13) when $\alpha=1 / 3, q=1 / 6, p=2 / 3, \beta=0.96$ and $b=1.052$. Such IFS is non-contractive, as $\lambda_{1}=2>1$, and its attractor is a generalized topological Cantor set, as the images of the two maps do not overlap because $f_{0}\left(\chi_{1}^{*}\right)<f_{1}\left(\chi_{0}^{*}\right)$.

While we are not aware of results establishing singularity vs. absolute continuity of the invariant measures for nonlinear IFS, Theorem 3 in Section 2 can be applied to provide a partial analysis of the affine IFS (15), which is summarized below. Note that such result is independent of the size of the shock $q$, the rate of time preference $\beta$ and the human capital productivity coefficient $b$.

Proposition 3 Let $\mu^{*}$ be the self-similar measure associated to the IFS (15)), $(\alpha \varphi, \alpha \varphi+(1-\alpha)$; $p,(1-p))$, on $[0,1]$.

i) If $0<\alpha<p^{p}(1-p)^{1-p}$, then $\mu^{*}$ is singular.

ii) If $\alpha=p^{p}(1-p)^{1-p}$ and $p \neq 1 / 2$, then $\mu^{*}$ is singular.

iii) If $\alpha=p=1 / 2$, then $\mu^{*}$ is absolutely continuous - it is the uniform (Lebesgue) measure over $[0,1]$.

iv) If $1 / 3 \leq p \leq 2 / 3$, then $\mu^{*}$ is absolutely continuous for Lebesgue a.e. $p^{p}(1-p)^{1-p}<\alpha<1$. 
v) If $0.156<p<1 / 3$ or $2 / 3<p<0.844$, then $\mu^{*}$ is absolutely continuous for Lebesgue a.e. $p^{p}(1-p)^{1-p}<\alpha<0.649$, while, for any $1<\gamma \leq 2$ such that $\left[p^{\gamma}+(1-p)^{\gamma}\right]^{1 /(\gamma-1)}<0.649$, $\mu^{*}$ has density in $L^{\gamma}$ for Lebesgue a.e. $\left[p^{\gamma}+(1-p)^{\gamma}\right]^{1 /(\gamma-1)} \leq \alpha<0.649$.

\section{A Three-Sector Model}

We now analyze an extended Uzawa-Lucas model, as presented in La Torre and Marsiglio (2010) and in Marsiglio (2012), where human capital needs to be endogenously allocated across three sectors: besides the physical and human capital sector as in the standard Uzawa-Lucas framework, also in the knowledge or technological sector 6 In this framework the planner has to choose consumption, $c_{t}$, and the share of human capital to allocate into physical, $u_{t}$, and knowledge, $v_{t}$, production, taking into account the presence of random shocks, which affect both physical and technological capital production. The technological knowledge, $a_{t}$, evolves over time because of newly produced knowledge: $a_{t+1}=y_{t}^{a}$. Both the production of the final consumption good, $y_{t}^{k}$, and knowledge, $y_{t}^{a}$, use a Cobb-Douglas technology. Physical production combines physical capital, knowledge capital and the allocated share of human capital: $y_{t}^{k}=k_{t}^{\alpha}\left(u_{t} h_{t}\right)^{\gamma} a_{t}^{1-\alpha-\gamma}$, with $0<\alpha<1$ and, $0<\gamma<1-\alpha$. Knowledge production combines the existing stock of knowledge and the allocated share of human capital: $y_{t}^{a}=\left(v_{t} h_{t}\right)^{1-\phi} a_{t}^{\phi}$, with $0<\phi<1$. The remaining share of human capital, $1-u_{t}-v_{t}>0,0<u_{t}, v_{t}<1$, is used to generate new human capital according to a linear technology (as in Section 3) : $h_{t+1}=b\left(1-u_{t}-v_{t}\right) h_{t}$, with $b>0$. We now assume that both the production of the final good and knowledge are affected by exogenous random shocks. As in the previous section, we keep assuming that educational choices are not affected by productivity shocks.

Specifically, the shocks are independent and identically distributed, and take on finite values: $z_{t}=\left\{q_{1}, q_{2}, 1\right\}$ and $\eta_{t} \in\{r, 1\}$, with $0<q_{1}<q_{2}<1$ and $0<r<1$, are the shocks affecting multiplicatively the physical and knowledge sector, respectively. As in La Torre et al. (2011), we assume that only three pairs of shock values can occur with positive probability, $\left(z_{t}, \eta_{t}\right) \in$ $\left\{\left(q_{1}, r\right),\left(q_{2}, 1\right),(1,1)\right\}$, each with (constant) probability $p_{0}, p_{1}$ and $p_{2}$ respectively, where $0<p_{i}<$ $1, i=0,1,2$, and $\sum_{i=0}^{2} p_{i}=1$. Such three shock configurations may be interpreted as: i) a deep economic-financial crisis having wide effects on the economy as a whole and thus involving both production and knowledge sectors, corresponding to $\left(z_{t}, \eta_{t}\right)=\left(q_{1}, r\right)$, ii) a negative supply shock, as an increase in raw materials' prices (e.g., oil), affecting only final production but not knowledge, corresponding to $\left(z_{t}, \eta_{t}\right)=\left(q_{2}, 1\right)$, and iii) a business-as-usual scenario, corresponding to $\left(z_{t}, \eta_{t}\right)=(1,1)$.

The social planner problem consists of choosing the level of consumption and the shares of human capital to allocate into physical and knowledge production in order to maximize social welfare, taking into account the dynamic evolution of physical, human and technological capital, the presence of random shocks $z_{t+1}=\left\{q_{1}, q_{2}, 1\right\}$ and $\eta_{t+1}=\{r, 1\}$, and the given initial conditions

\footnotetext{
${ }^{6}$ Note that referring to this sector as technological sector or another form of sector is totally irrelevant for our analysis. What really matters is that the third sector produces a form of capital (different from human and physical capital) which is used in the production of the final consumption good and which is produced using a certain share of human capital. Interpreting this kind of capital as cultural or social capital would work as well; however, in the following, for the sake of simplicity, we refer to this type of capital as technological or knowledge capital as in La Torre and Marsiglio (2010).
} 
$k_{0}, h_{0}, a_{0}, z_{0}$ and $\eta_{0}$ :

$$
\begin{gathered}
\quad V\left(k_{0}, h_{0}, a_{0}, z_{0}, \eta_{0}\right)=\max _{\left\{c_{t}, u_{t}, v_{t}\right\}_{t=0}^{\infty}} \mathbb{E}_{0} \sum_{t=0}^{\infty} \beta^{t} \ln c_{t} \\
\text { s.t. }\left\{\begin{array}{l}
k_{t+1}=z_{t} k_{t}^{\alpha}\left(u_{t} h_{t}\right)^{\gamma} a_{t}^{1-\alpha-\gamma}-c_{t} \\
h_{t+1}=b\left(1-u_{t}-v_{t}\right) h_{t} \\
a_{t+1}=\eta_{t}\left(v_{t} h_{t}\right)^{1-\phi} a_{t}^{\phi} \\
k_{0}>0, h_{0}>0, a_{0}>0, z_{0} \in\left\{q_{1}, q_{2}, 1\right\}, \eta_{0} \in\{r, 1\} \text { are given, }
\end{array}\right.
\end{gathered}
$$

where $\mathbb{E}_{0}$ denotes expectation at time $t=0$. The Bellman equation associated to (17) is:

$$
V\left(k_{t}, h_{t}, a_{t}, z_{t}, \eta_{t}\right)=\max _{c_{t}, u_{t}, v_{t}}\left[\ln c_{t}+\beta \mathbb{E}_{t} V\left(k_{t+1}, h_{t+1}, a_{t+1}, z_{t+1}, \eta_{t+1}\right)\right]
$$

Following the same steps used in the previous section, it is possible to prove the following.

Proposition 4 Given the problem in (17) under (18), the following results hold.

i) The Bellman equation (19) has a solution given by:

$$
V\left(k_{t}, h_{t}, a_{t}, z_{t}, \eta_{t}\right)=\theta+\theta_{k} \ln k_{t}+\theta_{h} \ln h_{t}+\theta_{a} \ln a_{t}+\theta_{z} \ln z_{t}+\theta_{\eta} \ln \eta_{t}
$$

where $\theta_{j}, j \in\{k, h, a, z, \eta\}$ are defined as follows:

$$
\begin{gathered}
\theta_{k}=\frac{\alpha}{1-\alpha \beta}, \quad \theta_{h}=\frac{\gamma(1-\beta)+\beta(1-\alpha)(1-\phi)}{(1-\alpha \beta)(1-\beta)(1-\beta \phi)}, \quad \theta_{a}=\frac{1-\alpha-\gamma}{(1-\alpha \beta)(1-\beta \phi)} \\
\theta_{z}=\frac{1}{1-\alpha \beta}, \quad \theta_{\eta}=\frac{(1-\alpha-\gamma) \beta}{(1-\alpha \beta)(1-\beta \phi)}
\end{gathered}
$$

and the constant term $\theta$ is given by:

$$
\begin{aligned}
\theta=\frac{1}{1-\beta}\{\ln (1-\alpha \beta) & +\frac{\gamma}{1-\alpha \beta} \ln \bar{u}+\frac{\alpha \beta}{1-\alpha \beta} \ln (\alpha \beta) \\
& +\frac{\beta \gamma(1-\beta)+\beta^{2}(1-\alpha)(1-\phi)}{(1-\alpha \beta)(1-\beta)(1-\beta \phi)} \ln [b(1-\bar{u}-\bar{v})] \\
+ & \left.\frac{\beta(1-\phi)(1-\alpha-\gamma)}{(1-\alpha \beta)(1-\beta \phi)} \ln \bar{v}+\frac{\beta}{1-\alpha \beta} \mathbb{E} \ln z+\frac{(1-\alpha-\gamma) \beta^{2}}{(1-\alpha \beta)(1-\beta \phi)} \mathbb{E} \ln \eta\right\},
\end{aligned}
$$

where $\bar{u}$ and $\bar{v}$ are defined in (20) and (21) respectively.

ii) The optimal policy rules for consumption, $c_{t}$, share of human capital allocated to physical, $u_{t}$, and knowledge, $v_{t}$, production are respectively given by:

$$
\begin{array}{ll}
c_{t}=(1-\alpha \beta) \bar{u}^{\gamma} z_{t} k_{t}^{\alpha} h_{t}^{\gamma} a_{t}^{1-\alpha-\gamma}, \\
u_{t} \equiv \frac{\gamma(1-\beta)(1-\beta \phi)}{\gamma(1-\beta)+\beta(1-\alpha)(1-\phi)}=\bar{u} & \forall t \\
v_{t} \equiv \frac{\beta(1-\beta)(1-\phi)(1-\alpha-\gamma)}{\gamma(1-\beta)+\beta(1-\alpha)(1-\phi)}=\bar{v} & \forall t .
\end{array}
$$


The optimal dynamics of physical, $k_{t+1}$, human, $h_{t+1}$, and technological, $a_{t+1}$, capital are the following:

$$
\begin{aligned}
& k_{t+1}=\alpha \beta \bar{u}^{\gamma} z_{t} k_{t}^{\alpha} h_{t}^{\gamma} a_{t}^{1-\alpha-\gamma}, \\
& h_{t+1}=b(1-\bar{u}-\bar{v}) h_{t}, \\
& a_{t+1}=\bar{v}^{1-\phi} \eta_{t} h_{t}^{1-\phi} a_{t}^{\phi} .
\end{aligned}
$$

The proof of Proposition 4 is a long and (algebraically) tedious extension of that of Proposition 1 in La Torre et al. (2011); hence, we omit it.

Because the human capital sector employs a linear technology, the economy may experience sustained long-run growth (La Torre and Marsiglio, 2010); specifically, whenever $b>1 /(1-\bar{u}-\bar{v})$ physical, human and technological capital grow without any bound according to (22), (23) and (24). Again, by introducing the physical to human capital, $\chi_{t}=k_{t} / h_{t}$, and the knowledge to human capital, $\omega_{t}=a_{t} / h_{t}$, ratio variables, we can recast the previous system into a two-dimensional nonlinear system converging to some compact trapping subset of $\mathbb{R}^{2}$, which may provide the basis for a stationary SBGP equilibrium:

$$
\left\{\begin{array}{l}
\chi_{t+1}=\Delta z_{t} \chi_{t}^{\alpha} \omega_{t}^{1-\alpha-\gamma} \\
\omega_{t+1}=\Theta \eta_{t} \omega_{t}^{\phi}
\end{array}\right.
$$

where

$$
\Delta=\frac{\alpha \beta \bar{u}^{\gamma}}{b(1-\bar{u}-\bar{v})} \quad \text { and } \quad \Theta=\frac{\bar{v}^{1-\phi}}{b(1-\bar{u}-\bar{v})}
$$

Under our assumptions on the stochastic process governing the exogenous shocks, the nonlinear IFS associated to (25) is defined by the following three maps $f_{i}: \mathbb{R}^{2} \rightarrow \mathbb{R}^{2}$ :

$$
\begin{cases}f_{0}(\chi, \omega)=\left(\Delta q_{1} \chi^{\alpha} \omega^{1-\alpha-\gamma}, \Theta r \omega^{\phi}\right) & \text { with probability } p_{0} \\ f_{1}(\chi, \omega)=\left(\Delta q_{2} \chi^{\alpha} \omega^{1-\alpha-\gamma}, \Theta \omega^{\phi}\right) & \text { with probability } p_{1} \\ f_{2}(\chi, \omega)=\left(\Delta \chi^{\alpha} \omega^{1-\alpha-\gamma}, \Theta \omega^{\phi}\right) & \text { with probability } p_{2}\end{cases}
$$

which has trapping set $A$ defined by the rectangle in $\mathbb{R}^{2}$ containing all three fixed points of each map in (27), that is, the rectangle with opposite vertex having coordinates

$$
\begin{aligned}
& \left(\chi_{0}^{*}, \omega_{0}^{*}\right)=\left(\left[\Delta q_{1}(\Theta r)^{(1-\alpha-\gamma) /(1-\phi)}\right]^{1 /(1-\alpha)},(\Theta r)^{1 /(1-\phi)}\right) \\
& \left(\chi_{2}^{*}, \omega_{2}^{*}\right)=\left(\left[\Delta \Theta^{(1-\alpha-\gamma) /(1-\phi)}\right]^{1 /(1-\alpha)}, \Theta^{1 /(1-\phi)}\right)
\end{aligned}
$$

such a scenario corresponds to growth rate values for the original variables, $k_{t}, h_{t}$ and $a_{t}$, oscillating between finite positive bounds. If the IFS (27) converges to some invariant measure supported over (possibly a fractal subset of) $A$, then we have a stationary SBGP equilibrium.

\subsection{Transforming Two-Dimensional Nonlinear IFS into Affine Ones}

Following the same approach pursued in Proposition 2, the next proposition shows that, for all feasible values for parameters $\alpha, \beta, \gamma, \phi, b, q_{1}, q_{2}, r$, a one-to-one logarithmic transformation exists such that (25) can be converted in a conjugate affine IFS converging to an invariant measure supported on a (possibly fractal) subset of a compact set. 
Proposition 5 The one-to-one logarithmic transformation $\left(\chi_{t}, \omega_{t}\right) \rightarrow\left(\varphi_{t}, \psi_{t}\right)$ defined by:

$$
\begin{aligned}
& \varphi_{t}=\rho_{1} \ln \chi_{t}+\rho_{2} \ln \omega_{t}+\rho_{3} \\
& \psi_{t}=\rho_{4} \ln \omega_{t}+\rho_{5},
\end{aligned}
$$

with

$$
\begin{gathered}
\rho_{1}=-\frac{1-\alpha}{\ln q_{2}}, \quad \rho_{2}=-\frac{(1-\alpha)\left(\ln q_{2}-\ln q_{1}\right)}{\ln q_{2} \ln r}, \quad \rho_{4}=-\frac{1-\phi}{\ln r}, \\
\rho_{3}=\left(1+\frac{\delta}{1-\alpha}\right)\left(1+\frac{\ln \Theta}{\ln r}\right)+\frac{\ln \Delta}{\ln q_{2}}-\frac{\ln q_{1} \ln \Theta}{\ln q_{2} \ln r},
\end{gathered}
$$

where

$$
\delta=\frac{1-\alpha}{(1-\phi) \ln q_{2}}\left[(1-\alpha-\gamma) \ln r+(\phi-\alpha)\left(\ln q_{2}-\ln q_{1}\right)\right]
$$

defines a contractive affine IFS which is equivalent to the nonlinear dynamics in (25) composed of three maps $w_{0}, w_{1}, w_{2}: \mathbb{R}^{2} \rightarrow \mathbb{R}^{2}$, given by:

$$
\begin{cases}w_{0}(\varphi, \psi)=(\alpha \varphi+\delta \psi, \phi \psi) & \text { with probability } p_{0} \\ w_{1}(\varphi, \psi)=(\alpha \varphi+\delta \psi, \phi \psi+(1-\phi)) & \text { with probability } p_{1} \\ w_{2}(\varphi, \psi)=(\alpha \varphi+\delta \psi+(1-\alpha), \phi \psi+(1-\phi)) & \text { with probability } p_{2}\end{cases}
$$

The IFS (34) converges in the Monge-Kantorovich metric to a unique self-similar measure supported on a compact attractor $A^{*} \subset \mathbb{R}^{2}$.

It is convenient to rewrite the IFS in (34) as

$$
\left\{\begin{array}{l}
\varphi_{t+1}=\alpha \varphi_{t}+\delta \psi_{t}+\zeta_{t} \\
\psi_{t+1}=\phi \psi_{t}+\vartheta_{t}
\end{array}\right.
$$

from which it can be seen that the (conjugate) random vector $\left(\zeta_{t}, \vartheta_{t}\right) \in \mathbb{R}^{2}$ taking on the three values $(0,0),(0,1-\phi)$ and $(1-\alpha, 1-\phi)$ corresponds respectively to the three scenarios $\left(q_{1}, r\right)$, $\left(q_{2}, 1\right)$ and $(1,1)$ for the original random values $\left(z_{t}, \eta_{t}\right)$.

Proof of Proposition 5. We use (29) and (30) to rewrite both sides of (35):

$$
\left\{\begin{array}{l}
\rho_{1} \ln \chi_{t+1}+\rho_{2} \ln \omega_{t+1}+\rho_{3}=\alpha\left(\rho_{1} \ln \chi_{t}+\rho_{2} \ln \omega_{t}+\rho_{3}\right)+\delta\left(\rho_{4} \ln \omega_{t}+\rho_{5}\right)+\zeta_{t} \\
\rho_{4} \ln \omega_{t+1}+\rho_{5}=\phi\left(\rho_{4} \ln \omega_{t}+\rho_{5}\right)+\vartheta_{t} .
\end{array}\right.
$$

Then, we use (25) to rewrite the LHS in each equation above so to obtain the following two equations:

$$
\begin{gathered}
\rho_{1} \ln \Delta+\rho_{1} \ln z_{t}+\rho_{1} \alpha \ln \chi_{t}+\rho_{1}(1-\alpha-\gamma) \ln \omega_{t}+\rho_{2} \ln \Theta+\rho_{2} \ln \eta_{t}+\rho_{2} \phi \ln \omega_{t}+\rho_{3} \\
=\alpha \rho_{1} \ln \chi_{t}+\alpha \rho_{2} \ln \omega_{t}+\alpha \rho_{3}+\delta \rho_{4} \ln \omega_{t}+\delta \rho_{5}+\zeta_{t}, \\
\rho_{4} \ln \Theta+\rho_{4} \ln \eta_{t}+\rho_{4} \phi \ln \omega_{t}+\rho_{5}=\phi \rho_{4} \ln \omega_{t}+\phi \rho_{5}+\vartheta_{t} .
\end{gathered}
$$

As the terms $\alpha \rho_{1} \ln \chi_{t}$ in (37) and $\phi \rho_{4} \ln \omega_{t}$ in (38) both cancel out, in order to let (37) hold independently of values taken by the variable $\ln \omega_{t}$, the following condition must hold:

$$
(1-\alpha-\gamma) \rho_{1}+\phi \rho_{2}=\alpha \rho_{2}+\delta \rho_{4}
$$


which, after substituting for $\rho_{1}, \rho_{2}$ and $\rho_{4}$ as in (31), boils down to (33).

Hence, when (33) holds equations (37) and (38) become

$$
\begin{gathered}
\rho_{1} \ln \Delta+\rho_{2} \ln \Theta+(1-\alpha) \rho_{3}-\delta \rho_{5}=\zeta_{t}-\rho_{1} \ln z_{t}-\rho_{2} \ln \eta_{t} \\
\rho_{4} \ln \Theta+(1-\phi) \rho_{5}=\vartheta_{t}-\rho_{4} \ln \eta_{t} .
\end{gathered}
$$

Note that we have arranged both equations so that the LHS are constant; therefore, both RHS in (39) and (40) must be constant as well for all the three-values configuration of the (corresponding) random shocks,

$$
\left(z_{t}, \eta_{t}, \zeta_{t}, \vartheta_{t}\right) \in\left\{\left(q_{1}, r, 0,0\right),\left(q_{2}, 1,0,1-\phi\right),(1,1,1-\alpha, 1-\phi)\right\},
$$

that is, the RHS in (39) and (40) must be independent of the random shocks values. Using the three values in (41) we get the following system of three equations [the first two out of the RHS in (39) and the last out of the RHS in (40)]:

$$
\left\{\begin{array}{l}
-\rho_{1} \ln q_{1}-\rho_{2} \ln r=-\rho_{1} \ln q_{2} \\
-\rho_{1} \ln q_{2}=1-\alpha \\
-\rho_{4} \ln r=1-\phi
\end{array}\right.
$$

which can be solved for $\rho_{1}, \rho_{2}$ and $\rho_{4}$, yielding the values in (31). As the RHS in (39) and (40) must be equal to $(1-\alpha)$ and $(1-\phi)$ respectively, $\rho_{3}$ and $\rho_{5}$ are computed by substituting the values of $\rho_{1}, \rho$ and $\rho_{4}$ in (31) into the LHS of (39) and (40):

$$
\left\{\begin{array}{l}
(1-\alpha) \rho_{3}-\delta \rho_{5}=(1-\alpha)\left[1+\frac{\ln \Delta}{\ln q_{2}}+\frac{\left(\ln q_{2}-\ln q_{1}\right) \ln \Theta}{\ln q_{2} \ln r}\right] \\
(1-\phi) \rho_{5}=(1-\phi)\left(1+\frac{\ln \Theta}{\ln r}\right)
\end{array}\right.
$$

which easily yield the values in (32).

Therefore, whenever $\delta$ is defined by (33), (37) and (38) are always satisfied for the values in (31) and (32), which is sufficient to establish that the affine IFS in (35) is topologically conjugate to the nonlinear IFS (25). To complete the proof, note that the upper triangular matrix defining the linear part of (35) has largest eigenvalue equals to $\max \{\alpha, \phi\}<1$, which implies that it is contractive; hence, Theorems 1 and 2 in Section 2 apply and the IFS (35) - or, equivalently, (34) - converges in the Monge-Kantorovich metric to a unique self-similar measure supported on a compact set $A^{*} \subset \mathbb{R}^{2}$.

Remark 2 As it happens for the IFS (13), also the nonlinear IFS (27) may be non-contractive, because there could exist a neighborhood of the smallest fixed point $\left(\chi_{0}^{*}, \omega_{0}^{*}\right)$ defined in (28) on which at least one of the two components of the largest map $f_{2}(\chi, \omega)$ in (27) has (maximum) slope steeper than 1, which, in turn, implies that its Lipschitz constant is larger than 1, $\lambda_{2}>$ 1. Again, in such circumstances the results of Section 2 cannot be directly applied; nonetheless, Proposition 5 establishes indirectly the existence of a unique invariant measure for (27) even in such non-contractive cases. Because, for any $i=0,1,2$, the nonlinear maps $f_{i}(\chi, \omega)$ in (27) have components which are strictly increasing and strictly concave, their Lipschitz constants $\lambda_{i}$ can be computed as the largest eigenvalue of their Jacobian matrix evaluated at the smallest fixed point $\left(\chi_{0}^{*}, \omega_{0}^{*}\right)$ defined in (28), which can be easily checked to be $\lambda_{0}=\max \{\alpha, \phi\}, \lambda_{1}=\max \left\{\alpha q_{2} / q_{1}, \phi / r\right\}$ and $\lambda_{2}=\max \left\{\alpha / q_{1}, \phi / r\right\}$ respectively. Clearly, under our assumptions it may well occur that 
either $\alpha / q_{1}>1$ or $\phi / r>1$ hold; if this is the case, the nonlinear IFS (27) turns out to be noncontractive, but, as Proposition 5 still applies, such IFS converges anyway to a unique invariant measure.

For example, this is certainly the case when we set $\alpha=1 / 3, \phi=0.8, \gamma=0.2, q_{1}=0.2$, $q_{2}=0.8, r=0.1, \beta=0.96$ and $b=1.052$ [so that there is sustained growth as $b>1.042=$ $1 /(1-\bar{u}-\bar{v})]$, because $\lambda_{2}=\max \left\{\alpha / q_{1}, \phi / r\right\}=\max \{1.667,8\}=8>1$. Furthermore, if we set $p_{0}=1 / 3$ and take any positive value $0<p_{1}<2 / 3$ so that $0<p_{2}=2 / 3-p_{1}<1$ holds al well, it turns out that the IFS (27) is not even 'contracting on average' according to (16). Indeed, noting that $\lambda_{0}=\max \{\alpha, \phi\}=\max \{1 / 3,0.8\}=0.8$ and $\lambda_{1}=\max \left\{\alpha q_{2} / q_{1}, \phi / r\right\}=\max \{1.333,8\}=8$, we have

$$
\sum_{i=0}^{2} p_{i} \ln \lambda_{i}=\frac{1}{3} \ln (0.8)+\left(p_{1}+p_{2}\right) \ln (8)=\frac{1}{3} \ln (0.8)+\frac{2}{3} \ln (8) \simeq 1.312>0 .
$$

\subsection{Generalized Sierpinski Gaskets as Attractors}

In this subsection we investigate the possibility of generating known fractals as attractors of the IFS (35); specifically, we focus our attention on Sierpinski gasket-like attractors. Let us rewrite the IFS (35) as

$$
\left[\begin{array}{l}
\varphi_{t+1} \\
\psi_{t+1}
\end{array}\right]=\left[\begin{array}{ll}
\alpha & \delta \\
0 & \phi
\end{array}\right]\left[\begin{array}{l}
\varphi_{t} \\
\psi_{t}
\end{array}\right]+\left[\begin{array}{l}
\zeta_{t} \\
\vartheta_{t}
\end{array}\right]
$$

where the vector $\left(\zeta_{t}, \vartheta_{t}\right) \in \mathbb{R}^{2}$ takes on the three values $(0,0),(0,1-\phi)$ and $(1-\alpha, 1-\phi)$. It is well known that, in order to generate a Sierpinski gasket-type attractor the linear part in (43) must be a diagonal matrix, i.e., $\delta=0$ must hold. Setting $\delta=0$ in condition (33) of Proposition 5 . implies that some restrictions on parameters must be imposed; specifically, the following relation among the random shocks values must be satisfied:

$$
r=\left(\frac{q_{2}}{q_{1}}\right)^{\frac{\alpha-\phi}{1-\alpha-\gamma}} .
$$

That is, one random shock value becomes a function of the other two; to ease reading, in (44) we set the value of the shock on knowledge production as a function of the two shocks affecting the final good production. Note that, in turn, this restriction implies that $\alpha<\phi$ must hold, as, because under our assumptions $q_{2} / q_{1}>1$ and $1-\alpha-\gamma>0$, this additional restriction is necessary to have $r<1$.

Corollary 2 If $\alpha<\phi$ and $r$ is defined according to (44), then the IFS (34)/(35) converges in the Monge-Kantorovich metric to a unique self-similar measure supported on a generalized Sierpinski gasket7 with vertices $(0,0),(0,1)$ and $(1,1)$.

Proof. When $\delta=0$ the three maps IFS (35) becomes

$$
\left\{\begin{array}{l}
\varphi_{t+1}=\alpha \varphi_{t}+\zeta_{t} \\
\psi_{t+1}=\phi \psi_{t}+\vartheta_{t}
\end{array}\right.
$$

${ }^{7}$ By generalized Sierpinski gasket we mean a standard Sierpinski gasket whose pre-fractals are composed by triangles that may or may not overlap, depending on the parameters configuration. 
which, when $\left(\zeta_{t}, \vartheta_{t}\right) \in\{(0,0),(0,1-\phi),(1-\alpha, 1-\phi)\}$, is well known to have a generalized Sierpinski gasket as attractor centered in the triangle having vertex the three fixed points of the maps in (45), $(0,0),(0,1)$ and $(1,1)$ respectively.

Note that the condition $\alpha<\phi$ required in Corollary 2 precludes the possibility of generating the standard, symmetric Sierpinski triangle with vertices $(0,0),(0,1)$ and $(1,1)$ through (35), as its construction postulates that $\alpha=\phi=1 / 2$ must hold. Hence, the attractor of (35) must always be a generalized Sierpinski gasket, that is, a Sierpinski gasket-like set whose prefractals 8 are composed by triangles that do not overlap for smaller values of $\alpha$ and $\phi$, while they tend to overlap for larger values of either $\alpha$ or $\phi$ (see the examples in Section 5).

Remark 3 Corollary 2 emphasizes the role of the coefficient $\delta$ defined in (33) in the proof of Proposition 5: it allows the transformation defined by (29) and (30) to have full control over all parameters' values. In other words, it acts as a correction factor affecting variable $\psi_{t}$ in (35) in such a way so to balance the effects induced by all model parameters (factor shares and exogenous shocks' values), letting the log-transformation always work, for any feasible parameters' values. Whenever, as in Corollary 2 , one puts some restrictions on the $\delta$ value, the range of applicability of Proposition 5 dramatically narrows, as heavy restrictions on parameters immediately become necessary in order to maintain the result of Proposition 5.

\subsection{Singular Self-similar Measures}

The IFS defined by (34)/(35) is two-dimensional, mapping $\mathbb{R}^{2}$ into itself; therefore, in order to pursue singularity vs. absolute continuity analysis on its self-similar measure we should rely on Theorem 4 of Section 2, Unfortunately, this theorem can be applied only to affine IFS which are similitudes according to condition (3), that is, if and only if they are defined through some orthogonal matrix $Q_{i}$ as in (44). As a matter of fact, the linear part of (35) is given by the upper triangular matrix

$$
Q=\left[\begin{array}{ll}
\alpha & \delta \\
0 & \phi
\end{array}\right]
$$

which precludes any application of this Theorem unless $\delta=0$ and $\alpha=\phi$, which, according to the discussion in Section 4.2, is incompatible with the assumptions on parameters' values for our model 9

However, by applying the main result of Section 2, Theorem 5, to the IFS (35) it is possible to establish the following sufficient condition for the singularity of the invariant measure. As there are three maps in the IFS (34), we can set only two out of the three probabilities associated to each map $w_{i}$, say $p_{0}$ and $p_{1}$, as the third must complement to $1: p_{2}=1-p_{0}-p_{1}$. Let

$$
E\left(p_{0}, p_{1}\right)=p_{0}^{p_{0}} p_{1}^{p_{1}}\left(1-p_{0}-p_{1}\right)^{1-p_{0}-p_{1}}
$$

denote the (exponential of the) entropy of the Bernoulli process underlying the exogenous shocks in our model.

Proposition 6 If $\alpha \phi<E\left(p_{0}, p_{1}\right)$, with $E\left(p_{0}, p_{1}\right)$ defined in $(46)$, then the invariant measure $\mu^{*}$ of the IFS (34)/(35) is singular.

\footnotetext{
${ }^{8}$ The sets obtained after each iteration of the map (34) are called prefractals.

${ }^{9}$ Note that, while on the one hand Theorem 4 is sufficiently general to encompass maps $w_{i}$ in the IFS with different contraction factors [which is not the case with (34)/(35)], on the other hand the requirement that they are similitudes implies that each map $w_{i}$ must apply the same contraction factor to both variables, $\varphi$ and $\psi$, as the contraction shrink must be the same along any direction in $\mathbb{R}^{2}$.
} 


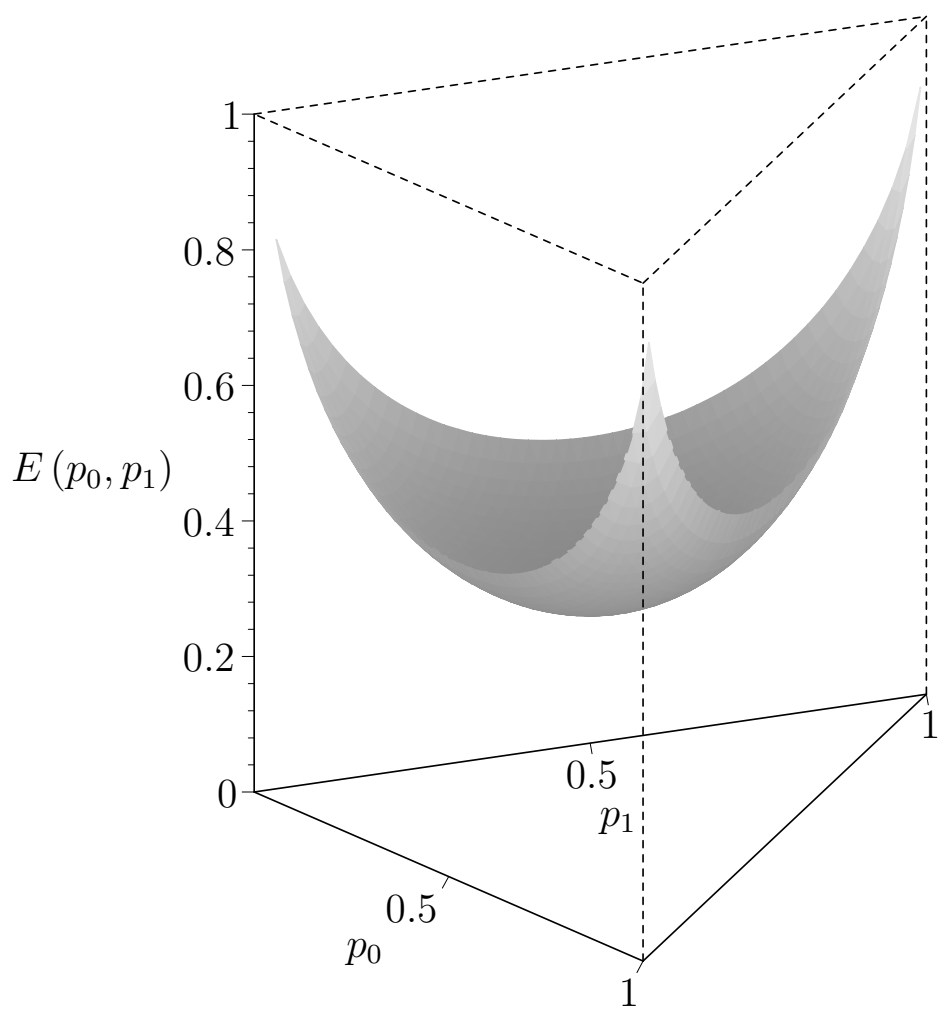

FIgURE 2: if $\alpha \phi<E\left(p_{0}, p_{1}\right)$ on the triangle $\left\{\left(p_{0}, p_{1}\right): p_{0} \geq 0, p_{1} \geq 0, p_{0}+p_{1} \leq 1\right\}$, then the invariant measure $\mu^{*}$ of the IFS (34) is singular.

Figure 2 illustrates Proposition 6, any value for the product $\alpha \phi$ below the entropy curve, i.e., satisfying $0<\alpha \phi<E\left(p_{0}, p_{1}\right)$, characterizes a dynamic defined by the IFS (34) that converges to a singular self-similar measure $\mu^{*}$. Nothing can be said on the possible absolute continuity of $\mu^{*}$ when $\alpha \phi \geq E\left(p_{0}, p_{1}\right)$. Note that when two out of the three probabilities $p_{i}$ are both close to 0 the invariant measure $\mu^{*}$ turns out to be singular for most values of $\alpha$ and $\phi$.

Consistently with Proposition [3, Proposition 6] states that singular invariant measures are associated with economies having small physical capital share in final good production $(\alpha)$; the novelty for the three-sector version is that the same effect can also be determined by a small knowledge share $(\phi)$, or, equivalently, a large human capital share in the knowledge sector $(1-\phi)$, while the human capital share in final production $(\gamma)$, does not affect the nature of the invariant measure. Again, such result is independent of the size of the shocks $q_{1}, q_{2}$ and $r$, the rate of time preference $\beta$ and the human capital productivity coefficient $b$. Note that empirical estimates of the physical capital share are about 1/3 (Bernanke and Gurkaynak, 2002); thus, in view of Propositions 3 and 6, empirically relevant values of $\alpha$ can be considered 'small'. While clear empirical estimates of the human capital share in knowledge production do not exist, it is reasonable to believe the technological sector to be human capital intensive (Lucas, 1988), such that relevant values of parameter $1-\phi$ should be considered 'large', or, equivalently, those of $\phi$ should be considered 'small'. Both these results go in the same direction to ensure that the inequality in Proposition 6 is met, meaning that for an empirically realistic model's parameterization the invariant measure $\mu^{*}$ is likely to be singular. 


\section{$5 \quad$ Examples}

Below we present some examples of attractors for certain parameterizations of the model described in Section 4. In all examples we keep constant the discount factor, $\beta=0.96$, and the random shocks' values on the final consumption good production, $q_{1}=0.2$ and $q_{2}=0.6$; moreover, we set $b=1 /(1-\bar{u}-\bar{v})+0.01$, where $\bar{u}$ and $\bar{v}$ are defined in (20) and (21) respectively, so to have always sustained growth. The first three examples cover the general setting envisaged by Proposition 5 for which we keep constant the random shock value on knowledge production, $r=0.5$, while the last three satisfy the restrictions of Corollary 2 so that $\alpha<\phi$ must hold and the $r$ value is constrained to be given by (44) - and thus the IFS (34) produces generalized Sierpinski gaskets as attractors.

Figure 3(a) shows a good estimate of the attractor of the affine IFS (34), obtained after 8 iterations of the operator (2) associated with it 10 starting from the triangle with vertices given by the fixed points of the three maps in (34):

$$
\left(\varphi_{0}^{*}, \psi_{0}^{*}\right)=(0,0), \quad\left(\varphi_{1}^{*}, \psi_{1}^{*}\right)=\left(\frac{\delta}{1-\alpha}, 1\right), \quad\left(\varphi_{2}^{*}, \psi_{2}^{*}\right)=\left(\frac{\delta}{1-\alpha}+1,1\right)
$$

where $\delta$ is given by (33), for $\alpha=\phi=1 / 2$ and $\gamma=0.1$. For such parameterization the 'correction factor' turns out to be $\delta=0.54277$, which, according to (47), implies that $\varphi_{1}^{*}=1.08553$ and $\varphi_{2}^{*}=2.08553$. Figure $3(\mathrm{~b})$ reports an estimate of the attractor associated to the nonlinear IFS (27) obtained after 8 iterations of the operator (2), which, according to Proposition 5, corresponds to the attractor in Figure 3(a) for the original nonlinear optimal dynamic described in (25). The latter iterations start from the triangle with vertices given by the fixed points of the three maps in (27), which, recalling (26), are computed as

$$
\begin{aligned}
& \left(\chi_{0}^{*}, \omega_{0}^{*}\right)=\left(\left[\Delta q_{1}(\Theta r)^{(1-\alpha-\gamma) /(1-\phi)}\right]^{1 /(1-\alpha)},(\Theta r)^{1 /(1-\phi)}\right)=(0.00007,0.00772) \\
& \left(\chi_{1}^{*}, \omega_{1}^{*}\right)=\left(\left[\Delta q_{2}(\Theta r)^{(1-\alpha-\gamma) /(1-\phi)}\right]^{1 /(1-\alpha)}, \Theta^{1 /(1-\phi)}\right)=(0.00194,0.03088) \\
& \left(\chi_{2}^{*}, \omega_{2}^{*}\right)=\left(\left[\Delta \Theta^{(1-\alpha-\gamma) /(1-\phi)}\right]^{1 /(1-\alpha)}, \Theta^{1 /(1-\phi)}\right)=(0.00540,0.03088) .
\end{aligned}
$$

As $\alpha \phi=1 / 4<1 / 3=\min \left\{E\left(p_{0}, p_{1}\right): p_{0} \geq 0, p_{1} \geq 0, p_{0}+p_{1} \leq 1\right\}=E(1 / 3,1 / 3)$, according to Proposition 6 the invariant measure $\mu^{*}$ supported over the attractor in Figure 3(a) must be singular for any choice of probabilities $p_{0}, p_{1}$.

Figure 4(a) shows the estimate of the attractor of the affine IFS (34), obtained through the same construction as before, for $\alpha=1 / 3, \phi=2 / 3$ and $\gamma=1 / 3$. In this case $\delta=-0.52916$, a negative value, which, according to (47), implies that $\varphi_{1}^{*}=-0.79374$, also negative, and $\varphi_{2}^{*}=0.20626$; in fact, the attractor lies across the first and second orthant. Figure 4(b) reports an estimate of the attractor of the corresponding nonlinear IFS (27). The three fixed points in the latter case now are

$$
\left(\chi_{0}^{*}, \omega_{0}^{*}\right)=(0.00011,0.00229), \quad\left(\chi_{1}^{*}, \omega_{1}^{*}\right)=(0.00163,0.01829), \quad\left(\chi_{2}^{*}, \omega_{2}^{*}\right)=(0.00351,0.01829) .
$$

Again, as $\alpha \phi=2 / 9<1 / 3$, the invariant measure $\mu^{*}$ supported over the generalized Sierpinski gasket in Figure 4(a) is singular for any choice of probabilities $p_{0}, p_{1}$.

\footnotetext{
${ }^{10}$ The Maple 18 code generating all attractor approximations in this section is available from the authors upon request.
} 


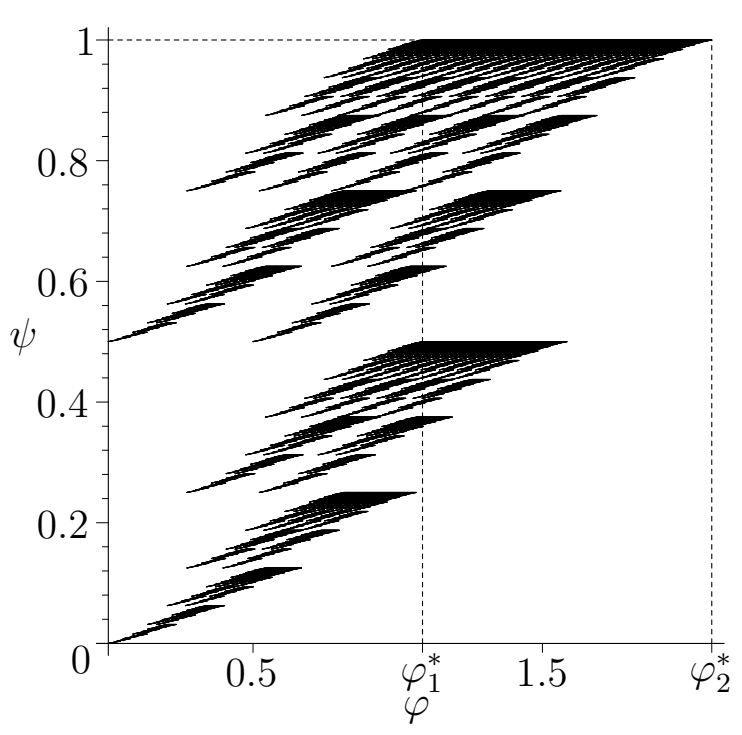

(a)

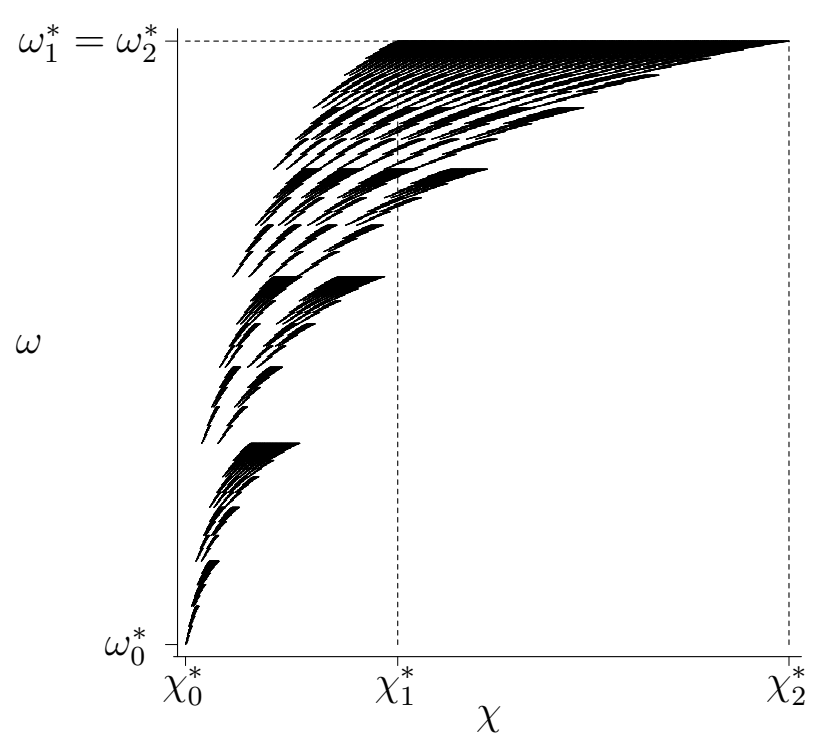

(b)

Figure 3: first 8 iterations of (a) the IFS (34) and (b) the IFS (27) for $\alpha=\phi=1 / 2$ and $\gamma=0.1$.

Figure 5(a) shows the estimate of the attractor of the affine IFS (34), again obtained through the same construction as before, for $\alpha=0.7, \phi=1 / 2$ and $\gamma=0.15$. Now $\delta=0.38020$, which, according to (47), implies that $\varphi_{1}^{*}=1.26734$ and $\varphi_{2}^{*}=2.26734$. Figure 5(b) reports the attractor of the corresponding nonlinear IFS (27). The fixed points in the latter case are

$$
\left(\chi_{0}^{*}, \omega_{0}^{*}\right)=(0.00001,0.00471), \quad\left(\chi_{1}^{*}, \omega_{1}^{*}\right)=(0.00093,0.01884), \quad\left(\chi_{2}^{*}, \omega_{2}^{*}\right)=(0.00510,0.01884) .
$$

Because now $\alpha \phi=0.35>1 / 3$, according to Proposition 6 we cannot exclude that the invariant measure $\mu^{*}$ supported over the attractor in Figure 5(a) may be absolutely continuous for some values of probabilities $p_{0}, p_{1}$, possibly for $p_{0}=p_{1}=1 / 3$ or values around $1 / 3$. In fact, both attractors in Figure 5 exhibit enough overlapping of the prefractals to allow for positive 2-dimensional Lebesgue measure of the attractor itself and an absolutely continuous invariant measure supported on it.

The last three examples deal with attractors which are generalized Sierpinski gasket according to Corollary 2; hence, from now on $\alpha<\phi$ will hold and, by (44),

$$
r=\left(\frac{q_{2}}{q_{1}}\right)^{\frac{\alpha-\phi}{1-\alpha-\gamma}}=3^{\frac{\alpha-\phi}{1-\alpha-\gamma}} .
$$

Now the fixed points of the three maps in (34) are always $\left(\varphi_{0}^{*}, \psi_{0}^{*}\right)=(0,0),\left(\varphi_{1}^{*}, \psi_{1}^{*}\right)=(0,1)$ and $\left(\varphi_{2}^{*}, \psi_{2}^{*}\right)=(1,1)$.

In Figure 6 the attractors of both the affine IFS (34) and the nonlinear IFS (27) are obtained through the usual procedure for $\alpha=1 / 2, \phi=0.501$ and $\gamma=0.4$, which, according to (48), imply that $r=0.98907$. Note that in Figure 6(a) the plot resembles the standard symmetric Sierpinski triangle; this occurs because, although $\alpha<\phi$, in this example we chose a value for $\phi$ very close to $\alpha=1 / 2$. The fixed points of the maps in the corresponding nonlinear IFS (27) in Figure 6(b)] are

$$
\left(\chi_{0}^{*}, \omega_{0}^{*}\right)=(0.00022,0.00720), \quad\left(\chi_{1}^{*}, \omega_{1}^{*}\right)=(0.00197,0.00736), \quad\left(\chi_{2}^{*}, \omega_{2}^{*}\right)=(0.00546,0.00736) .
$$

As $\alpha \phi=0.2505<1 / 3$, also in this case the invariant measure $\mu^{*}$ supported over the attractor in Figure 6(a) is singular for any choice of probabilities $p_{0}, p_{1}$. 


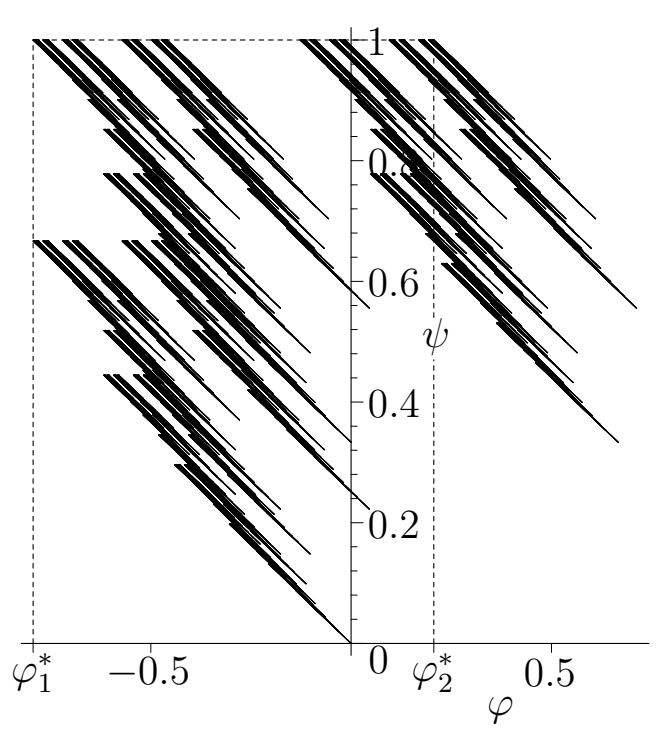

(a)

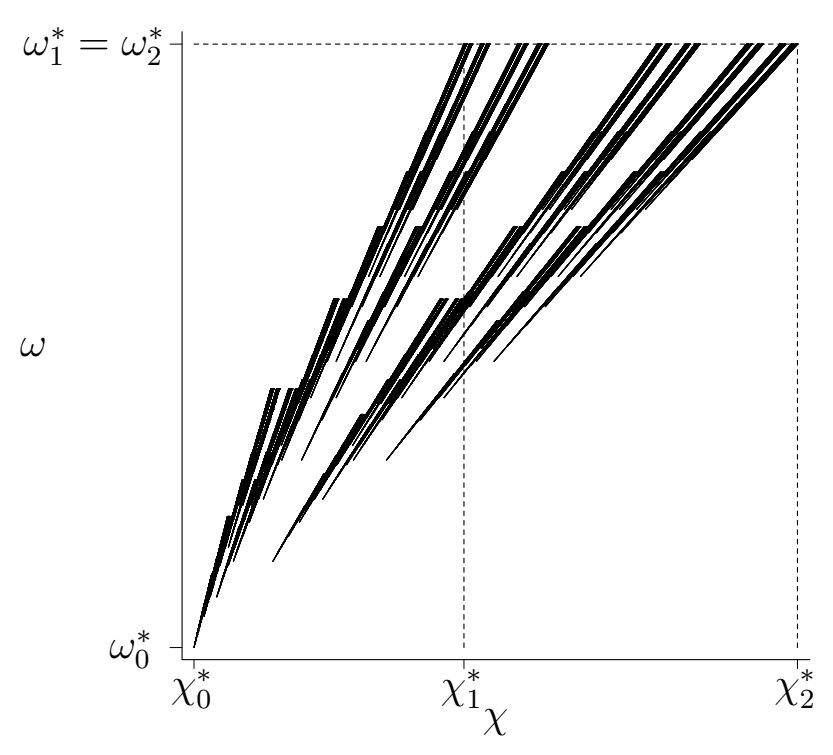

(b)

FiguRE 4: first 8 iterations of (a) the IFS (34) and (b) the IFS (27) for $\alpha=1 / 3, \phi=2 / 3$ and $\gamma=1 / 3$.

Remark 4 Attractors similar to those in Figure [6] can be constructed outside the range of application of Corollary 2 by setting $\alpha=\phi=1 / 2$, provided that a value for $\gamma$ sufficiently close to $1-\alpha=1 / 2$ is chosen. Indeed, if $\alpha=\phi$ and $\gamma \simeq 1-\alpha$, the value of $\delta$ in (33) is close to $0-e . g$., if $\gamma=0.499$ and all other parameters' values are as in the first example, $\delta=0.00136$-yielding an IFS (34)/(35) with attractor resembling the standard symmetric Sierpinski triangle.

Note that if one sets $\gamma=1-\alpha=1 / 2$, the attractor becomes the exact standard symmetric Sierpinski triangle; however, when substituted into (18) or into (25), the condition $\gamma=1-\alpha$ implies the disappearance of the knowledge sector in our model. In other words, the only way to obtain a symmetric Sierpinski gasket as the attractor of a three-sector growth model is by eliminating one of the sectors, thus annihilating the very nature of the model itself, transforming it into the two-dimensional one discussed in Section 3, which, after detrending its two state variables $k_{t}$ and $h_{t}$, exhibits a one-dimensional dynamic possibly converging to a Cantor set. In this case, the lack of the 'correction factor' $\delta$ let the two logarithmic transformations defined by (29) -(30) in Proposition 5 become linearly dependent, 11 thus ruling out any chance of keeping the twodimensional nature of the original dynamic (25) through the transformation, as it necessarily collapses into the one-dimensional dynamic (15) tackled in Section 3.

In Figure 7 we consider an example with smaller values of both $\alpha$ and $\phi$ : specifically, we consider $\alpha=0.4, \phi=0.45$ and $\gamma=0.3$. From (48) we get $r=0.83268$. The fixed points of the three maps in (27) in Figure 7(b) are

$$
\left(\chi_{0}^{*}, \omega_{0}^{*}\right)=(0.00023,0.01358), \quad\left(\chi_{1}^{*}, \omega_{1}^{*}\right)=(0.00169,0.01894), \quad\left(\chi_{2}^{*}, \omega_{2}^{*}\right)=(0.00396,0.01894) .
$$

Clearly, as $\alpha \phi=0.18<1 / 3$, the invariant measure $\mu^{*}$ supported over the attractor in Figure $7(\mathrm{a})$ is singular for any choice of probabilities $p_{0}, p_{1}$. This property is confirmed by the strong no-overlapping of the prefractals in both Figures 7(a) and 7(b).

Finally, Figure 8 reports an example with larger values of both $\alpha$ and $\phi: \alpha=0.6$ and $\phi=0.65$ and $\gamma=0.2$, so that, according to (48), $r=0.75984$. The fixed points of the three maps in (27)

\footnotetext{
${ }^{11}$ We thank L. Gardini for raising this observation during the MDEF 2014 workshop.
} 


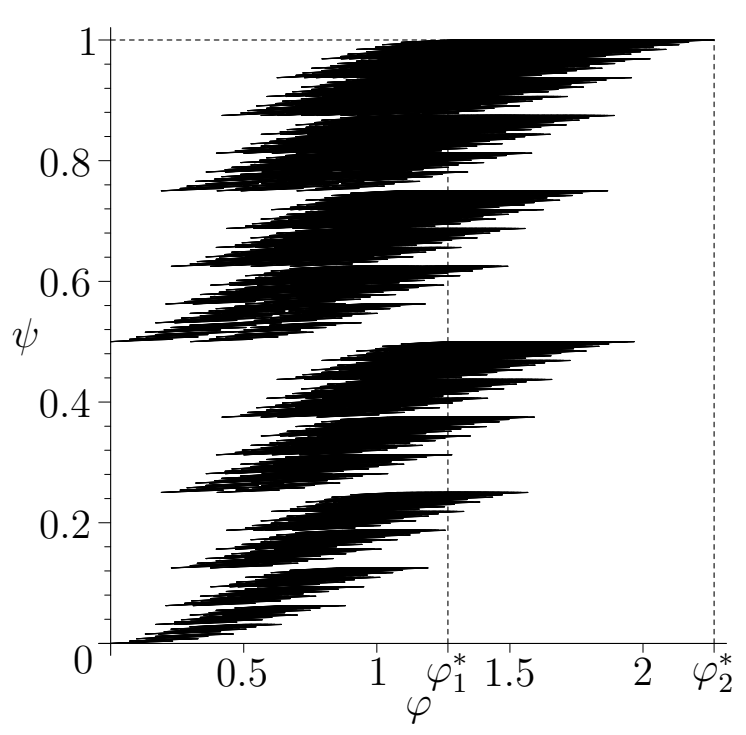

(a)

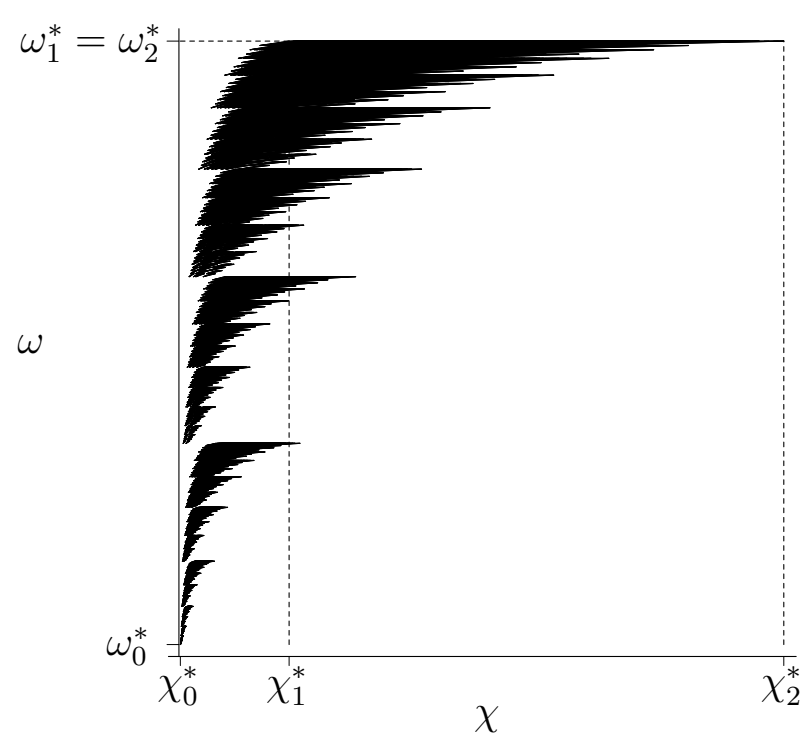

(b)

FiguRE 5: first 8 iterations of (a) the IFS (34) and (b) the IFS (27) for $\alpha=0.7, \phi=1 / 2$ and $\gamma=0.15$.

in Figure 8(b) are

$$
\left(\chi_{0}^{*}, \omega_{0}^{*}\right)=(0.00006,0.00838), \quad\left(\chi_{1}^{*}, \omega_{1}^{*}\right)=(0.00135,0.01837), \quad\left(\chi_{2}^{*}, \omega_{2}^{*}\right)=(0.00484,0.01837) .
$$

In this case $\alpha \phi=0.39>1 / 3$, so that, according to Proposition 6, an absolutely continuous invariant measure $\mu^{*}$ supported over the attractor in Figure 8(a) cannot be ruled out for some values of probabilities $p_{0}, p_{1}$, possibly for $p_{0}=p_{1}=1 / 3$. As a matter of fact, both attractors in Figure 8 exhibit enough overlapping of the prefractals so that the attractor may have positive Lebesgue measure, possibly with an absolutely continuous invariant measure supported on it.

\section{Conclusions}

In this paper we extend the analysis of stochastic discrete-time optimal growth models to consider the multi-sectoral framework in the context of sustained growth. We consider first the simplest case, namely the Uzawa-Lucas (1988) two-sector model, and then an extended three-sector model, as in La Torre and Marsiglio (2010). Both the models exhibit two peculiar features: the log-CobbDouglas structure of preferences and production functions in each sector allows for a closed form solution of the Bellman equation, thus permitting to explicitly compute the optimal dynamics of the state variables; moreover, through simple log-transformations of the capital ratio variable dynamics we are able to show that the model economy converges to an invariant measure supported on some compact set which, under some restrictions on parameters, may exhibit a fractal nature (a generalized Cantor set in the case of the two-sector model, and a generalized Sierpinski gasket in the case of the three-sector model).

By exploiting some recent results on the IFS theory, we are also able to establish some sufficient conditions under which the invariant measure turns out to be either singular or absolute continuous with respect to Lebesgue measure (for the three-sector model we only consider the singularity with respect to Lebesgue measure). By comparing the outcomes of the two and three-sector model, it is clear that the latter framework is much richer and allows for a greater variety of alternative 


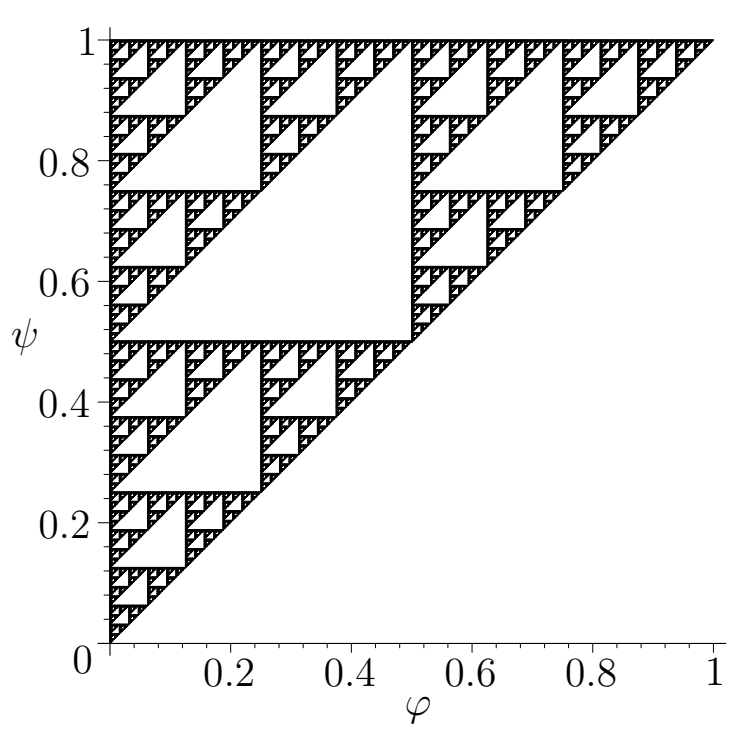

(a)

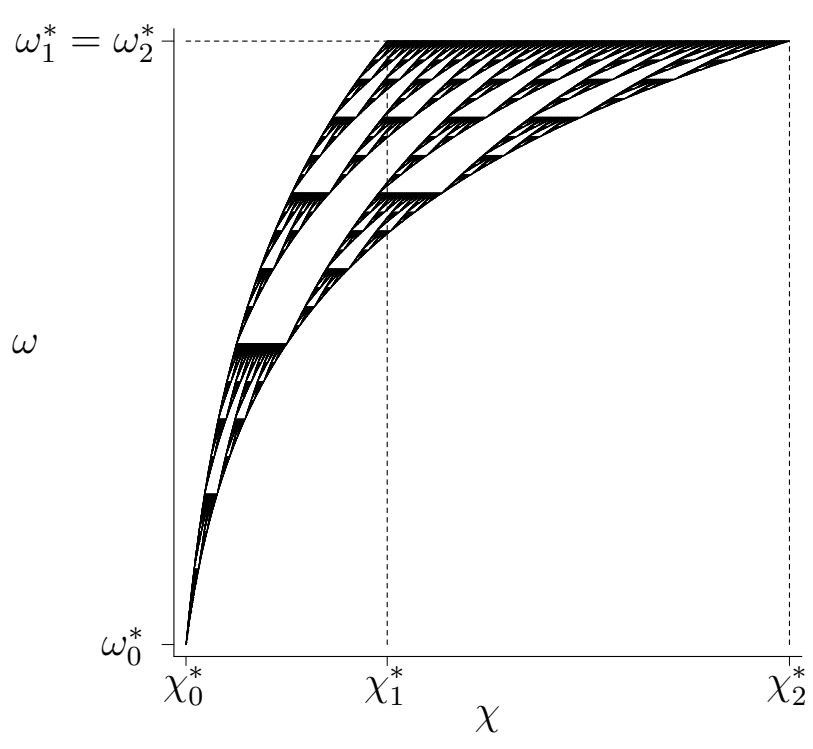

(b)

FiguRE 6: first 8 iterations of (a) the IFS (34) and (b) the IFS (27) for $\alpha=1 / 2, \phi=0.501, \gamma=0.4$ and $r=0.98907$.

configurations in terms of singularity and absolute continuity of the self-similar measure associated to the IFS. We are also able to show that, in both models, for a rich range of parameter value (also for an empirically realistic model's parameterization) the invariant measure is likely to be singular.

This paper significantly extends the literature on stochastic growth and fractal attractors, by showing that also stochastic BGP equilibria can have a fractal nature and showing that for empirically relevant parameterizations the invariant measure is singular. Despite the new insights provided by these results, new questions for future research naturally arise. In particular, since in Section 4 we are able only to comment on the singularity of the invariant measure, the issue related to its eventual absolute continuity is still unsolved; it would be interesting at least to build an example converging to some absolutely continuous invariant measure. Moreover, since the literature has only focused thus far on log-linear transformations of the optimal dynamics associated with stochastic growth models (the same approach we use in our paper), it might be interesting to try characterizing the singularity vs. absolute continuity of the invariant measure directly for the original nonlinear IFS - i.e., without transforming them into affine IFS - in both two and three-sector models. These issues are left for future research.

\section{References}

[1] Barnsley MF (1989). Fractals everywhere. New York: Academic Press.

[2] Barnsley MF, Demko S (1985). Iterated function systems and the global construction of fractals. Proc Roy Soc London Ser A 399: 243-275.

[3] Barnsley MF, Hutchinson J, Stenflo O (2008). V-variable fractals: fractals with partial self similarity. Advances in Math 218: 2051-2088. 


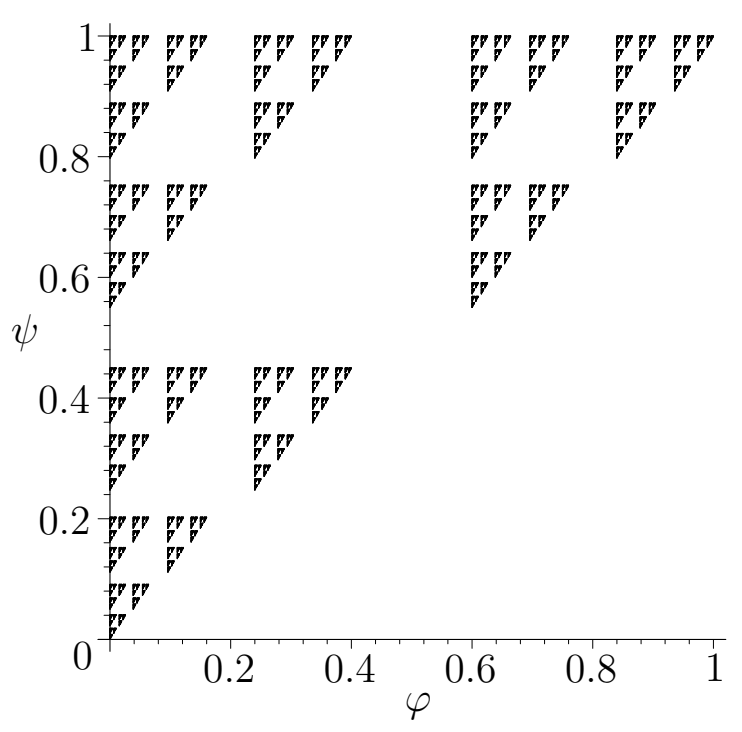

(a)

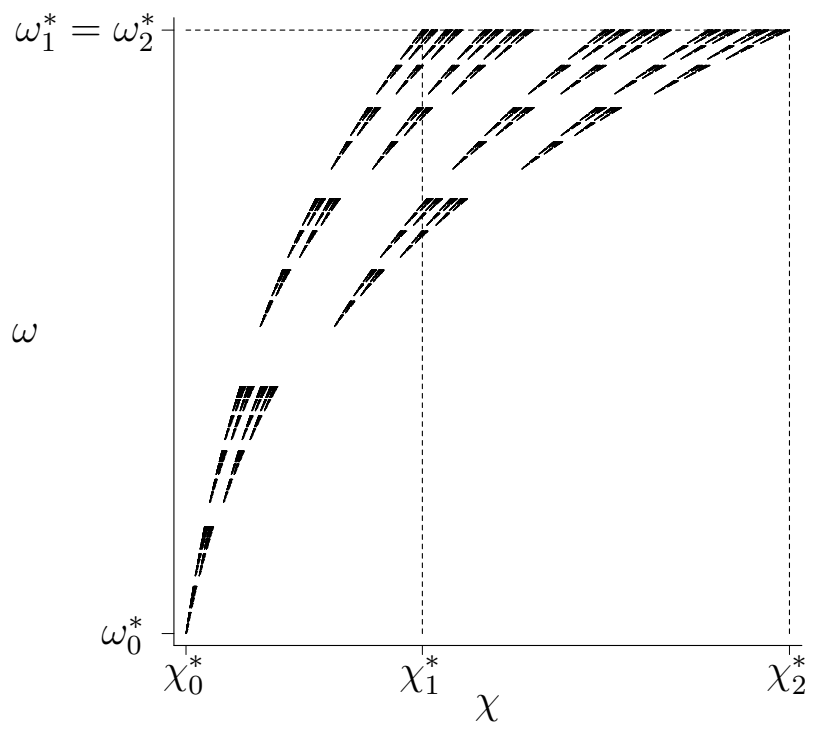

(b)

FiguRE 7: first 8 iterations of (a) the IFS (34) and (b) the IFS (27) for $\alpha=0.4, \phi=0.45, \gamma=0.3$ and $r=0.83268$.

[4] Bernanke BS, Gurkaynak RS (2002). Is growth exogenous? Taking Mankiw, Romer, and Weil seriously. In: Bernanke BS, Rogoff K, eds. NBER Macroeconomics Annual 16. Cambridge: MIT Press, 11-57.

[5] Bethmann D (2007). A closed-form solution of the Uzawa-Lucas model of endogenous growth. J Econ 90: 87-107.

[6] Bethmann D (2013). Solving Macroeconomic Models with Homogeneous Technology and Logarithmic Preferences. Australian Economics Papers 52 (1): 1-18.

[7] Bischi GI, Chiarella C, Kopel M, Szidarovszky F (2010). Nonlinear oligopolies - stability and bifurcations. Berlin Heidelberg: Springer-Verlag.

[8] Boldrin M, Montrucchio L (1986). On the indeterminacy of capital accumulation paths. J Econ Theor 40: 26-39.

[9] Brock WA, Hommes CH (1997). A rational route to randomness. Econometrica 65: 10591095.

[10] Brock WA, Mirman LJ (1972). Optimal economic growth and uncertainty: the discounted case. J Econ Theory 4: 479-513.

[11] Diaconis D, Freedman P (1999). Iterated Random Functions. SIAM Review 41: 45-76.

[12] Falconer KJ, Miao J (2007). Dimensions of self-affine fractals and multifractals generated by upper-triangular matrices. Fractals 15: 289-299.

[13] Freiberg UR, La Torre D, Mendivil F (2011). Iterated function systems and stability of variational problems on self-similar objects. Nonlinear Anal R World Appl 12: 1123-1129. 


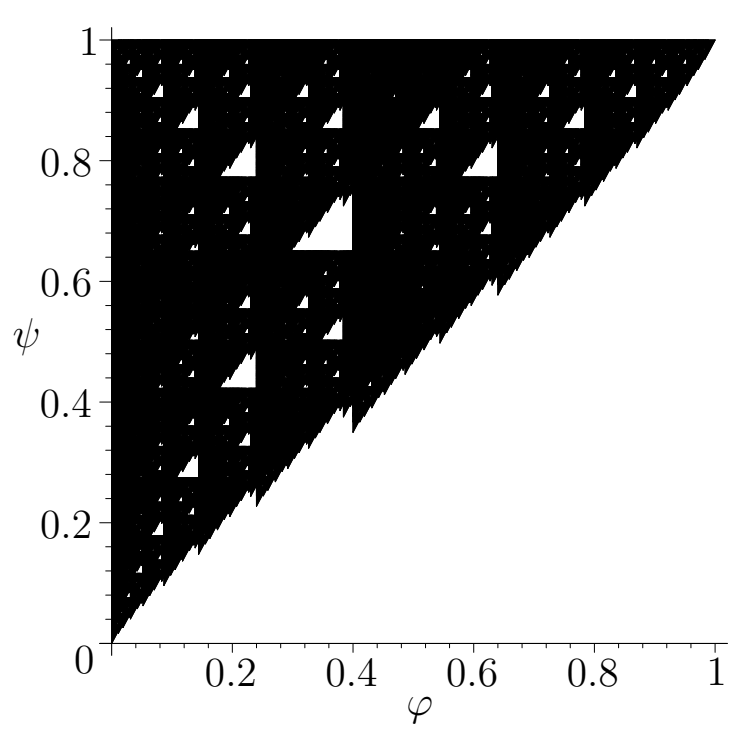

(a)

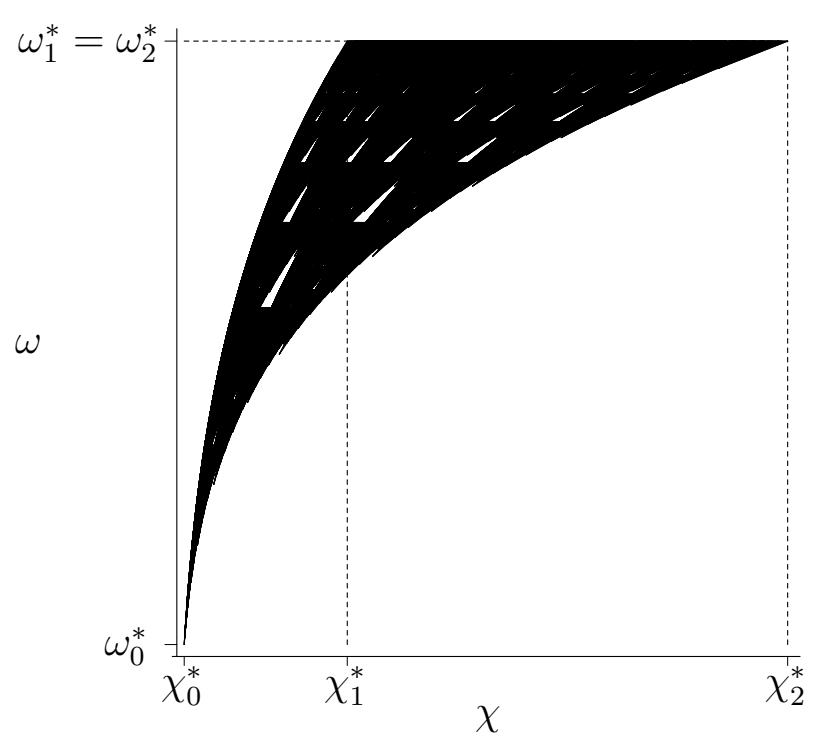

(b)

Figure 8: first 8 iterations of (a) the IFS (34) and (b) the IFS (27) for $\alpha=0.6, \phi=0.65, \gamma=0.2$ and $r=0.75984$.

[14] Gardini L, Hommes CH, Tramontana F, de Vilder R (2009). Forward and backward dynamics in implicitly defined overlapping generations models. J Econ Behav Organ 71: 110-129.

[15] Hutchinson J (1981). Fractals and self-similarity. Indiana Univ J Math 30: 713-747.

[16] Kunze H, La Torre D, Vrscay ER (2007). Contractive multifunctions, fixed point inclusions and iterated multifunction systems. J Math Anal Appl 330: 159-173.

[17] Iacus SM, La Torre D (2005a). A comparative simulation study on the IFS distribution function estimator. Nonlinear Anal R World Appl 6: 858-873.

[18] Iacus SM, La Torre D (2005b). Approximating distribution functions by iterated function systems. J Appl Math Decis Sci 1: 33-46.

[19] La Torre D, Marsiglio S (2010). Endogenous technological progress in a multi-sector growth model, Economic Modelling 27: 1017-1028.

[20] La Torre D, Marsiglio S, Privileggi F (2011). Fractals and self-similarity in economics: the case of a two-sector growth model. Image Analysis \& Stereology 30: 143-151.

[21] La Torre D, Mendivil F, Vrscay ER (2006). IFS on multifunctions. In: Aletti G, Burger M, Micheletti A, Morale D, eds. Math everywhere: deterministic and stochastic modelling in biomedicine, economics and industry. Berlin Heidelberg: Springer-Verlag, 125-134.

[22] La Torre D, Mendivil F (2008). Iterated function systems on multifunctions and inverse problems. J Math Anal Appl 340: 1469-1479.

[23] La Torre D, Mendivil F (2009). Union-additive multimeasures and self-similarity. Commun Math Anal 7: 51-61. 
[24] La Torre D, Vrscay ER (2009). A generalized fractal transform for measure-valued images. Nonlinear Anal 71: e1598-e1607.

[25] La Torre D, Vrscay ER, Ebrahimi M, Barnsley MF (2009). Measure-valued images, associated fractal transforms, and the affine self-similarity of images. SIAM J Imaging Sci 2: 470-507.

[26] Lucas RE (1988). On the mechanics of economic development. J Monetary Econ 22: 3-42.

[27] Mandelbrot BB (1982). The fractal geometry of nature. San Francisco: Freeman.

[28] Mankiw GN, Romer D, Weil DN (1992). A Contribution to the Empirics of Economic Growth. Q J Econ 107: 407-437.

[29] Marsiglio S (2012). Economic growth: technical progress, population dynamics and sustainability, Rivista Italiana degli Economisti 17: 151-158.

[30] Marsiglio S (2012). Stochastic shocks in a two-sector Solow model. Int J Mathematical Modelling and Numerical Optimisation 3: 313-318.

[31] Mendivil F, Vrscay ER (2002a). Fractal vector measures and vector calculus on planar fractal domains. Chaos Solitons Fractals 14: 1239-1254.

[32] Mendivil F, Vrscay ER (2002b). Self-affine vector measures and vector calculus on fractals. In: Michael F, Barnsley MF, Saupe D, Vrscay ER, eds. Fractals in multimedia. Proceedings of the Meeting on Fractals in Multimedia. IMA Vol Math Appl 132. New York: Springer-Verlag, $137-155$.

[33] Mitra T, Montrucchio L, Privileggi F (2003). The nature of the steady state in models of optimal growth under uncertainty. Econ Theor 23: 39-71.

[34] Mitra T, Privileggi F (2004). Cantor type invariant distributions in the theory of optimal growth under uncertainty. J Difference Equ Appl 10: 489-500.

[35] Mitra T, Privileggi F (2006). Cantor type attractors in stochastic growth models. Chaos, Solitons Fractals 29: 626-637.

[36] Mitra T, Privileggi F (2009). On Lipschitz continuity of the Iterated Function System in a stochastic optimal growth model. J Math Econ 45: 185-198.

[37] Montrucchio L (1994). Dynamic complexity of optimal paths and discount factors for strongly concave problems. J Optim Theor Appl 80: 385-406.

[38] Montrucchio L, Privileggi F (1999). Fractal steady states in stochastic optimal control models. Ann Oper Res 88: 183-197.

[39] Ngai SM, Wang Y. (2005). Self-similar measures associated to IFS with non-uniform contraction ratios. Asian J. Math. 9: 227-244.

[40] Nishimura K, Sorger G (1996). Optimal cycles and chaos: A survey. Stud Nonlinear Dynam Econometrics 1: 11-28.

[41] Nishimura K, Yano M (1995). Nonlinear dynamics and chaos in optimal growth: an example. Econometrica 63: 981-1001. 
[42] Niu M, Xi L-F (2007). Singularity of a Class of Self-similar Measures. Chaos, Solitons Fractals 34: $376-382$.

[43] Peres Y, Schlag W (2000). Smoothness of Projections, Bernoulli Convolutions and the Dimension of Exceptions. Duke Mathematical Journal 102: 193-251.

[44] Peres Y, Schlag W, Solomyak B (2000). Sixty Years of Bernoulli Convolutions. In Bandt C, Graf S, Zahle M, eds. Fractal Geometry and Stochastics II. Basel: Birkhäuser Verlag, 39-65.

[45] Peres Y, Solomyak B (1996). Absolute Continuity of Bernoulli Convolutions, a Simple Proof. Mathematical Research Letters 3: 231-239.

[46] Peres Y, Solomyak B (1998). Self-Similar Measures and Intersections of Cantor Sets. Transactions of the American Mathematical Society 350: 4065-4087.

[47] Privileggi F, Marsiglio S (2013). Environmental shocks and sustainability in a basic economyenvironment model. Int J Applied Nonlinear Science 1: 67-75.

[48] Ramsey F (1928). A mathematical theory of saving, Econ J 38: 543-559.

[49] Rebelo S (1991). Long-run policy analysis and long-run growth. J Polit Econ 99: 500-521.

[50] Roman S (1992). Coding and Information Theory. Graduate Texts in Mathematics 134. New York: Springer-Verlag.

[51] Schief A (1994). Separation Properties for Self-Similar Sets. Proceedings of the American Mathematical Society 122: 111-115.

[52] Shmerkin P (2006). Overlapping self-affine sets. Indiana Univ. Math. J. 55 4: 1291-1331.

[53] Solomyak B (1995). On the Random Series $\sum \pm \lambda^{n}$ (an Erdös Problem). Annals of Mathematics 142: 611-625.

[54] Stokey NL, Lucas RE (1989). Recursive methods in economic dynamics. Cambridge: Harvard University Press.

[55] Uzawa H (1965). Optimum technical change in an aggregate model of economic growth, Int Econ Rev 6: 18-31.

[56] Vrscay ER (1991). Moment and collage methods for the inverse problem of fractal construction with iterated function systems. In: Peitgen HO, Henriques JM, Penedo LF, eds. Fractals in the fundamental and applied sciences. North-Holland, 443-461. 